OPEN ACCESS

Edited by:

Isotta Chimenti,

Sapienza University of Rome, Italy

Reviewed by:

Subrata Chakrabarti,

Western University, Canada

Swayam Prakash Srivastava,

Yale University, United States

*Correspondence:

Gheyath K. Nasrallah

gheyath.nasrallah@qu.edu.qa

Arduino Aleksander Mangoni

arduino.mangoni@flinders.edu.au

Gianfranco Pintus

gpintus@sharjah.ac.ae

Specialty section:

This article was submitted to

Molecular Medicine,

a section of the journal

Frontiers in Cell and Developmental

Biology

Received: 21 March 2021

Accepted: 26 April 2021

Published: 19 May 2021

Citation:

Giordo R, Ahmed YMA, Allam H,

Abusnana S, Pappalardo L,

Nasrallah GK, Mangoni AA and

Pintus G (2021) EndMT Regulation by

Small RNAs in Diabetes-Associated

Fibrotic Conditions: Potential Link

With Oxidative Stress.

Front. Cell Dev. Biol. 9:683594.

doi: 10.3389/fcell.2021.683594

\section{EndMT Regulation by Small RNAs in Diabetes-Associated Fibrotic Conditions: Potential Link With Oxidative Stress}

\author{
Roberta Giordo1, Yusra M. A. Ahmed1, Hilda Allam", Salah Abusnana, ${ }^{2,3}$ \\ Lucia Pappalardo4, Gheyath K. Nasrallah" ${ }^{5,6 *}$, Arduino Aleksander Mangoni,8* and \\ Gianfranco Pintus ${ }^{1,9 *}$
}

\begin{abstract}
${ }^{1}$ Department of Medical Laboratory Sciences, College of Health Sciences and Sharjah Institute for Medical Research, University of Sharjah, Sharjah, United Arab Emirates, ${ }^{2}$ Department of Diabetes and Endocrinology, University Hospital Sharjah, Sharjah, United Arab Emirates, ${ }^{3}$ Department of Clinical Sciences, College of Medicine, University of Sharjah, Sharjah, United Arab Emirates, ${ }^{4}$ Department of Biology, Chemistry and Environmental Studies, American University of Sharjah, Sharjah, United Arab Emirates, ${ }^{5}$ Department of Biomedical Sciences, College of Health Sciences Member of QU Health, Qatar University, Doha, Qatar, ${ }^{6}$ Biomedical Research Center, Qatar University, Doha, Qatar, ${ }^{7}$ Discipline of Clinical Pharmacology, College of Medicine and Public Health, Flinders University, Adelaide, SA, Australia, ${ }^{8}$ Flinders Medical Centre, Adelaide, SA, Australia, ${ }^{9}$ Department of Biomedical Sciences, University of Sassari, Sassari, Italy
\end{abstract}

Diabetes-associated complications, such as retinopathy, nephropathy, cardiomyopathy, and atherosclerosis, the main consequences of long-term hyperglycemia, often lead to organ dysfunction, disability, and increased mortality. A common denominator of these complications is the myofibroblast-driven excessive deposition of extracellular matrix proteins. Although fibroblast appears to be the primary source of myofibroblasts, other cells, including endothelial cells, can generate myofibroblasts through a process known as endothelial to mesenchymal transition (EndMT). During EndMT, endothelial cells lose their typical phenotype to acquire mesenchymal features, characterized by the development of invasive and migratory abilities as well as the expression of typical mesenchymal products such as $\alpha$-smooth muscle actin and type I collagen. EndMT is involved in many chronic and fibrotic diseases and appears to be regulated by complex molecular mechanisms and different signaling pathways. Recent evidence suggests that small RNAs, in particular microRNAs (miRNAs) and long non-coding RNAs (IncRNAs), are crucial mediators of EndMT. Furthermore, EndMT and miRNAs are both affected by oxidative stress, another key player in the pathophysiology of diabetic fibrotic complications. In this review, we provide an overview of the primary redox signals underpinning the diabetic-associated fibrotic process. Then, we discuss the current knowledge on the role of small RNAs in the regulation of EndMT in diabetic retinopathy, nephropathy, cardiomyopathy, and atherosclerosis and highlight potential links between oxidative stress and the dyad small RNAs-EndMT in driving these pathological states.

Keywords: EndMT, miRNAs, diabetes, fibrosis, oxidative stress 


\section{INTRODUCTION}

Diabetes mellitus (DM) is one of the most common chronic diseases worldwide (Lin X. et al., 2020). A prediction study estimated a significant further increase in the number of people suffering from diabetes, especially in developing countries, with a global prevalence of $7.7 \%$ (439 million adults) by 2030 (Shaw et al., 2010; Lin X. et al., 2020). Long-term hyperglycemia is the main driver of the onset and the progression of common diabetic complications, particularly those affecting the eye, kidney, nervous system, and cardiovascular system (Deshpande et al., 2008). Such complications are secondary to structural and functional alterations of organs and tissues that are caused by an increased cellular glucose uptake (Wellen and Hotamisligil, 2005). This activates inflammatory pathways which ultimately leads to excessive deposition of extra cellular matrix (ECM) proteins and consequent thickening of the vessel wall (Wellen and Hotamisligil, 2005; Wynn, 2008). Tissue fibrosis is therefore the common denominator of most diabetic complications, including atherosclerosis, cardiomyopathy, nephropathy and retinopathy (Ban and Twigg, 2008). Myofibroblasts are the key mediators of pathological ECM accumulation (Kendall and Feghali-Bostwick, 2014). These cells are normally involved in tissue repair and are subsequently removed by apoptosis at the end of the repair process. However, under pathological situations, their unrestrained activation leads to excessive ECM deposition (Micallef et al., 2012). Myofibroblasts originate from different precursor cells, depending on the organ and the type of initial injury (Bochaton-Piallat et al., 2016). Although fibroblasts represent the primary source of myofibroblasts, the latter can also originate from the inresident or bone marrowderived mesenchymal cells as well as epithelial and endothelial cells (ECs), through a process known as epithelial/endothelial to mesenchymal transition (Micallef et al., 2012; Kendall and Feghali-Bostwick, 2014). In particular, endothelial to mesenchymal transition (EndMT), the process involving ECs, is emerging as an important player in the pathogenesis of diabetic fibrosis (Srivastava et al., 2013; Cao et al., 2014; Souilhol et al., 2018). ECs, constituting the inner layer of blood vessels, are responsible for maintaining vascular homeostasis in response to endogenous and exogenous perturbations (Sandoo et al., 2010; Khaddaj Mallat et al., 2017). There is good evidence that ECs, when exposed to hyperglycemia, undergo significant alterations that result in an imbalance between vasodilation and vasoconstriction as well as the development of inflammatory and vascular complications (Bakker et al., 2009; Meza et al., 2019). Moreover, high glucose concentrations have been shown to trigger the shift of the endothelium toward the mesenchymal phenotype (Yu et al., 2017; Giordo et al., 2021). Overall, EndMT appears to represent the key link in the interaction between inflammation and endothelial dysfunction in diabetic complications (Cho et al., 2018; Man et al., 2019). In the setting of EndMT, ECs lose their typical cobblestone morphology and tight junctions and acquire increased motility and the ability to secrete ECM proteins (Dejana et al., 2017). In addition, concurrently with the loss of typical endothelial markers, such as vascular endothelial cadherin (VE-cadherin), platelet endothelial cell adhesion molecule (PECAM-1), also known as CD31, and von Willebrand Factor (vWF), they acquire the ability to express several mesenchymal markers, such as alpha-smooth muscle actin ( $\alpha$-SMA), smooth muscle protein 22 alpha (SM22 $\alpha$ ), fibronectin, vimentin, and fibroblast specific protein-1 (FSP-1) (Dejana et al., 2017; Hong et al., 2018). EndMT is involved in many chronic and fibrotic disease states and appears to be regulated by several factors (Evrard et al., 2016; Thuan et al., 2018; Phan et al., 2020). In diabetes, oxidative stress is emerging as an important trigger of the ECs transformation into myofibroblasts and vascular remodeling (Montorfano et al., 2014; Thuan et al., 2018). Indeed, hyperglycemia can increase the production of reactive oxygen species (ROS), which in turn activate signaling pathways leading to the disruption of ECs hemostasis (Russell et al., 2002; Peng et al., 2013; Li et al., 2017; Volpe et al., 2018). Several signaling pathways have been demonstrated to be involved in EndMT regulation, e.g., transforming growth factor-beta (TGF- $\beta$ ) signaling, Notch signaling, fibroblast growth factor/fibroblast growth factor receptor 1 (FGF/FGFR1) signaling pathway, Smad2/3-mediated pathways (Piera-Velazquez and Jimenez, 2019) and pro-inflammatory signaling cascades (Lin et al., 2018; Ferreira et al., 2019). An important role in the regulation of EndMT is also played by micro RNAs (miRNAs), a class of short endogenous non-coding RNAs that regulate gene expression at post-transcriptional level by binding to the $3^{\prime}$ untranslated region of messenger RNA (mRNA) (Kim et al., 2015; Michlewski and Cáceres, 2019). A single miRNA can target multiple mRNAs, thus influencing several processes such as cell differentiation, proliferation, and apoptosis (Vidigal and Ventura, 2015). miRNAs can also target significant parts of pathways since miRNAs with similar (seed) sequence target similar sets of genes and thus similar sets of pathways (Kehl et al., 2017). Moreover miRNAs can, either positively or negatively, regulate gene expression (Catalanotto et al., 2016). As a result, they represent promising markers and druggable targets for many diseases, including diabetes (Regazzi, 2018; Cao et al., 2019; Fan et al., 2020). An increasing amount of evidence also suggests that diabetes progression is linked to the alteration of miRNAs expression profiles; indeed, profibrotic miRNAs, such as miR125b, let-7c, let-7g, miR-21, miR-30b, and miR-195 have been shown to be upregulated in EndMT. By contrast, antifibrotic miRNAs, such as miR-122a, miR-127, miR-196, and miR-375, with inhibitory action toward genes responsible for EndMT, have been shown to be downregulated (Ghosh et al., 2012; Kim, 2018; Srivastava et al., 2019). In addition to miRNAs, recent studies have also demonstrated the involvement of another class of small RNAs, known as long non-coding RNAs (lncRNAs), in diabetesassociated EndMT (Feng et al., 2017; Leung and Natarajan, 2018). Compared to miRNAs, the concentrations of lncRNAs are almost tenfold lower, with the latter exhibiting significant tissue and cell specificity (Cabili et al., 2011). However, the knowledge of the function and the regulation of IncRNAs are still limited. This review aims to summarize and discuss the available knowledge on the role of small RNAs in the regulation of EndMT in diabetes-associated fibrotic complications such as retinopathy, nephropathy, cardiomyopathy, atherosclerosis, and its potential link with oxidative. 


\section{DIABETIC NEPHROPATHY}

Diabetic nephropathy (DN) is the leading cause of chronic kidney disease in about $40 \%$ of patients with type 1 and type 2 diabetes (Gross et al., 2005). Poorly controlled blood glucose concentrations can damage the filtering functionality of the kidneys, which become unable to remove waste products and extra fluids from the body (Rheinberger and Böger, 2014; RuizOrtega et al., 2020). The symptoms of DN do not generally manifest in the early stages, but rather when kidney function has significantly deteriorated (Lim, 2014). Therefore, a tight blood glucose control is key to prevent the onset and progression of DN (Lewis and Maxwell, 2014; Ruiz-Ortega et al., 2020). The progression of DN is defined by various clinical stages which reflect the gradual involvement of tissue damage to different kidney compartments: glomerulus, tubules, vasculature and interstitium (Mogensen et al., 1983). The final stage of DN is characterized by renal fibrosis and organ failure, which are the result of the excessive accumulation of ECM (Calle and Hotter, 2020). Renal fibrosis is driven by multiple mechanisms, including glucose metabolism abnormalities associated with oxidative stress, inflammatory processes, and hemodynamic changes (Brosius, 2008). Consequently, many signaling pathways and cell types (mesangial cells, endothelial cells and podocytes) are involved in the fibrotic process (Badal and Danesh, 2014; Aghadavoud et al., 2017). As mentioned above, alterations of glucose metabolism not only activate various signaling pathways (Badal and Danesh, 2014; Aghadavoud et al., 2017) but also induce oxidative stress, a key pathophysiological step in the onset and progression of diabetes-associated vascular complications (Kashihara et al., 2010; Mima, 2013; Oguntibeju, 2019). Indeed, high glucose concentrations activate the diacylglycerol-protein kinase C (DAG-PKC) pathway, which is associated with endothelial dysfunction, increased production of extracellular matrix and activation of cytokines and transforming growth factor- $\beta$ (TGF- $\beta$ ) (Koya and King, 1998; Evcimen and King, 2007). In addition, protein kinase $C$ (PKC) induces oxidative stress by activating mitochondrial NADPH oxidase (Chen et al., 2014; Giordo et al., 2021). Increased glucose can also activate aldose reductase and the polyol pathway, leading to the depletion of Nicotinamide Adenine Dinucleotide Phosphate $(\mathrm{NADPH})$, which is also required for the generation of the cellular antioxidant nitric oxide (NO) (Tesfamariam, 1994; Hummel et al., 2006; Ying, 2008; Zhao et al., 2008). The reduced NO availability compromises the balance between ROS generation and antioxidant defense, one of the leading causes of endothelial dysfunction (Schiffrin, 2008). Furthermore, hyperglycemia enhances the formation of advanced glycation end products (AGEs), proteins or lipids that become glycated as a result of exposure to sugars (Goldin et al., 2006). AGEs increase ROS production and promote inflammation and fibrosis through the activation of PKC, the nuclear factor kappa light chain enhancer of activated B cells (NF-kB) and TGF- $\beta$ (Aghadavoud et al., 2017; Rhee and Kim, 2018). Within the hemodynamic factors driving renal fibrosis, an important role is played by the over-activation of the renin-angiotensin-aldosterone system (RAAS), a crucial hormone system in blood pressure regulation and fluid balance (Benigni et al., 2010; Patel et al., 2017). Hyperglycemia and insulin resistance increases the release of angiotensin II (Ang II) a potent vasoconstrictor belonging to the RAAS system (Giacchetti et al., 2005; Benigni et al., 2010; Williams and Scholey, 2018). Angiotensin II plays an important role in renal fibrosis by activating a number of factors responsible for ECM production such as TGF- $\beta$, PKC and NFКB (Badal and Danesh, 2014; Aghadavoud et al., 2017). On the other hand, Angiotensin-converting enzyme2 (ACE2), the main modulator of the RAAS system (Benigni et al., 2010), prevents the accumulation of Ang II by catalyzing the conversion of Ang II into the vasodilator Angiotensin I (Ang I) (Batlle et al., 2010; Williams and Scholey, 2018). Although no cure is available for $\mathrm{DN}$, the control of blood sugar levels and blood pressure, together with a healthy lifestyle, can slow or stop its progression. The most common DN treatments are based on the RAAS system inactivation; precisely with the use of either the ACE inhibitors (ACEis) or angiotensin receptor blockers (ARBs) or their combination (Anand and Tamura, 2012; Pathak and Dass, 2015). This type of treatments allows the lowering of proteinuria and the blood pressure within the glomerular capillaries. In addition, ACEis can also ameliorates kidney fibrosis in combination with other drugs. Is this the case of $N$-acetyl-seryl-aspartyl-lysyl-proline (AcSDKP) an antifibrotic peptide that, in combination with the ACEi, imidapril, improves kidney fibrosis restoring antifibrotic miRNAs, such as miR-29 and miR-let-7 and increasing the inhibition of the profibrotic dipeptidyl peptidase-4 (DPP-4) (Nitta et al., 2016; Srivastava et al., 2020a). DPP-4 inhibitors are another class of medicines used for DN's treatment. In this context, due to the highest affinity for DPP-4, the drug Linagliptin is one of the most widely used (Kanasaki, 2018). In addition, promising data also come from treatments aiming at restoring Sirtuin 3 (SIRT3), which appear to ameliorate renal damage, via inhibition of aberrant glycolysis and preserving mitochondrial homeostasis (Srivastava et al., 2018; Locatelli et al., 2020).

\section{miRNAs REGULATION OF DN-ASSOCIATED EndMT}

The ECM is a three-dimensional network of macromolecules (proteoglycans and fibrous proteins), present in all tissues and organs, that contributes to tissue morphogenesis, differentiation and homeostasis. Collagens, elastins, fibronectins, and laminins are the main proteins constituting the ECM (Frantz et al., 2010; Yue, 2014). The excessive deposition of ECM components is the hallmark of fibrosis, which represents a key pathophysiological step in many chronic inflammatory diseases, including diabetes (Herrera et al., 2018). Myofibroblasts are the main cellular mediators of fibrosis as they have the ability to invade the interstitial space and produce excessive amounts of ECM proteins (Zent and Guo, 2018). Although resident mesenchymal cells are the main source of myofibroblasts, the latter can also derive from other type of cells including pericytes, fibrocytes, epithelial and endothelial cells (ECs). The process involving ECs, known as EndMT, has been shown to actively contribute to 
the progression of renal fibrosis (Zeisberg et al., 2008; Curci et al., 2014; Sun et al., 2016). Besides, the mesenchymal shift contribution to kidney fibrosis can also be accelerate by the crosstalk between endothelium and epithelium, since EndMT can influence and induce EMT in tubular cells (Li et al., 2020b). In this context, $\mathrm{N}$-acetyl-seryl-aspartyl-lysyl-proline (AcSDKP) plays a crucial role in inhibiting both EndMT and EndMTmediated EMT. Its inhibitory action is exerted by targeting the fibroblast growth factor receptor 1 (FGFR1), an antifibrotic endothelial receptor ( $\mathrm{Li}$ et al., 2020b), and by controlling the metabolic switch between glucose and fatty acid metabolism. Indeed, defects in normal kidney metabolism can accelerate EndMT and EndMT-mediated EMT contributing to kidney fibrosis (Srivastava et al., 2018, 2020b). An increasing body of evidence suggests that miRNAs are key regulators of EndMT as they appear differentially expressed under fibrotic stimuli such as high glucose, TGF $\beta$, and hypoxia (Glover et al., 2019). This differential expression also reflects the specific role, profibrotic or antifibrotic, played by miRNAs (Hulshoff et al., 2019; Srivastava et al., 2019). The most potent inducer of kidney fibrosis is TGF- $\beta$ (Wang J. et al., 2016; Wang Z. et al., 2017), which can trigger EndMT either by activation of specific signaling pathways, such as Akt and Smad (Wang J. et al., 2016; Wang Z. et al., 2017), or by increasing the expression of pro-fibrotic miRNAs (Srivastava et al., 2019). In this context, TGF- $\beta$ mediates EndMT through the up-regulation of miR-21, a key modulator of fibrosis (Srivastava et al., 2013; Huang et al., 2015). Specifically, TGF- $\beta$ elicits miR-21 increase through the activation of Smad3 which regulates miR-21 expression both at a transcriptional and a post-transcriptional level (Zhong et al., 2011). In addition, Smad3 modulates the expression of other miRNAs and activates the expression of various fibrotic genes (Loboda et al., 2016). Another mechanism used by miR-21 to stimulate renal fibrosis is the inhibition of Smad7 protein, a negative regulator of TGF$\beta 1 /$ Smad3 signaling. In this context, Smad7 has been shown to suppress renal fibrosis by down-regulating pro-fibrotic miRNAs such as miR-21 and miR-192 while up-regulating the antifibrotic miR-29b (Chung et al., 2013; Loboda et al., 2016). Additionally, miR-21 also regulates TGF- $\beta$-mediated EndMT through the PTEN/Akt pathway (Kumarswamy et al., 2012). Specifically, TGF- $\beta$ increases the endothelial expression of miR21, which in turn decreases the expression of PTEN, ultimately promoting EndMT by Akt activation (Meadows et al., 2009; Medici et al., 2011; Kumarswamy et al., 2012). Another molecule linked to TGF- $\beta$ signaling in kidney fibrosis is the dipeptidyl peptidase-4 (DDP-4), a multi-functional protein expressed on the surface of most cell types, including ECs (Deacon, 2019). DPP4 overexpression induces TGF- $\beta$-mediated EndMT in diabetic nephropathy (Shi et al., 2015; Kanasaki, 2016). Furthermore, recent studies have reported a relationship between DPP-4 and miR-29 in diabetic kidney fibrosis, where the overexpression of DPP-4 results associated with the suppression of miR-29s family anti-fibrotic activity (Kriegel et al., 2012; Harmanci et al., 2017). In line with these observations, the use of the DPP-4 inhibitor, linagliptin, ameliorates kidney fibrosis by restoring miR-29s and consequentially inhibiting EndMT in diabetic mice (Kanasaki et al., 2014). The anti-fibrotic peptide, AcSDKP which suppresses the TGF- $\beta$-induced EndMT in diabetic kidney (Nagai et al., 2014; Hrenak et al., 2015) can also, alone or in combination with angiotensin-converting enzyme inhibitor (ACEi), ameliorates renal fibrosis by suppressing DPP-4 and restoring the antifibrotic miR-29s and miR-let-7s expression in TGF- $\beta$-induced EndMT (Srivastava et al., 2020a). The crosstalk between miR29s and miR-let-7s is crucial for maintaining endothelial cell homeostasis and AcSDKP potentiates this crosstalk regulation (Srivastava et al., 2019). Indeed, the presence of AcSDKP upregulates the antifibrotic miR-let-7 families, especially miRlet-7b, which suppress TGF $\beta$ R1 and TGF $\beta$ signaling (Srivastava et al., 2016). Suppression of TGF $\beta$ signaling results in the upregulation of the miR-29 family expression, which in turn induce FGFR1 phosphorylation, a critical step for miR-let-7 production (Srivastava et al., 2016, 2019). The associated expression of miR29 and miR-let-7 is also regulated by an alternative mechanism involving interferon-gamma (IFN $\gamma$ ) (Srivastava et al., 2019). Precisely, miR-29 target the profibrotic IFN $\gamma$ (Ma et al., 2011) blocking its inhibitory action toward FGFR1 which in turn induces the expression of miR-let-7 (Chen et al., 2012; Srivastava et al., 2019). Although not strictly related to DN, an additional anti-fibrotic mechanism, occurring by the suppression of DPP4, involves miR-448-3p. EndMT inhibition and amelioration of vascular dysfunction has been indeed observed in both diabetic mice and cell models overexpressing miR-448-3p (Guan et al., 2020). A further regulatory mechanism of EndMT in diabetic nephropathy involves miR-497 and its two targets, ROCK1 and ROCK2, which belong to the rho-associated kinases (ROCKs) family and are activated in diabetes (Kolavennu et al., 2008; Liu et al., 2018; Matoba et al., 2020). A recent study showed that ROCKs inhibition, following treatment with melatonin ( $N$-acetyl-5-methoxytryptamine), suppressed TGF- $\beta 2$-induced EndMT. Specifically, the negative modulation of ROCK1 and ROCK2 is associated with the melatonin-induced up-regulation of miR-497, both in glomerular cells and diabetic rats (Liu et al., 2018). See figures and associated tables to overview of the signaling pathways involving both anti-fibrotic (Figure $\mathbf{1}$ and Table 1) and pro-fibrotic (Figure 2 and Table 2) miRNAs.

\section{DIABETIC CARDIOMYOPATHY}

Diabetic cardiomyopathy (DCM), another common complication in diabetes, refers to myocardial dysfunction in the absence of conventional cardiovascular complications (coronary artery disease, valvular disease) and risk factors (hypertension, dyslipidemia) (Boudina and Abel, 2010; Jia et al., 2018). In the early stages, DCM is usually asymptomatic and characterized by left ventricular (LV) hypertrophy, LV diastolic dysfunction with diastolic filling abnormalities, myocardial fibrosis and cell signaling abnormalities. Disease progression leads to systolic dysfunction (left ventricular low ejection fraction) accompanied by heart failure, which is characterized by marked hypertrophy and fibrosis in the advanced stages (Boudina and Abel, 2010; Jia et al., 2018; Tan et al., 2020). Hyperglycemia, insulin resistance, lipid metabolism defects and oxidative stress up-regulate the production of advanced 


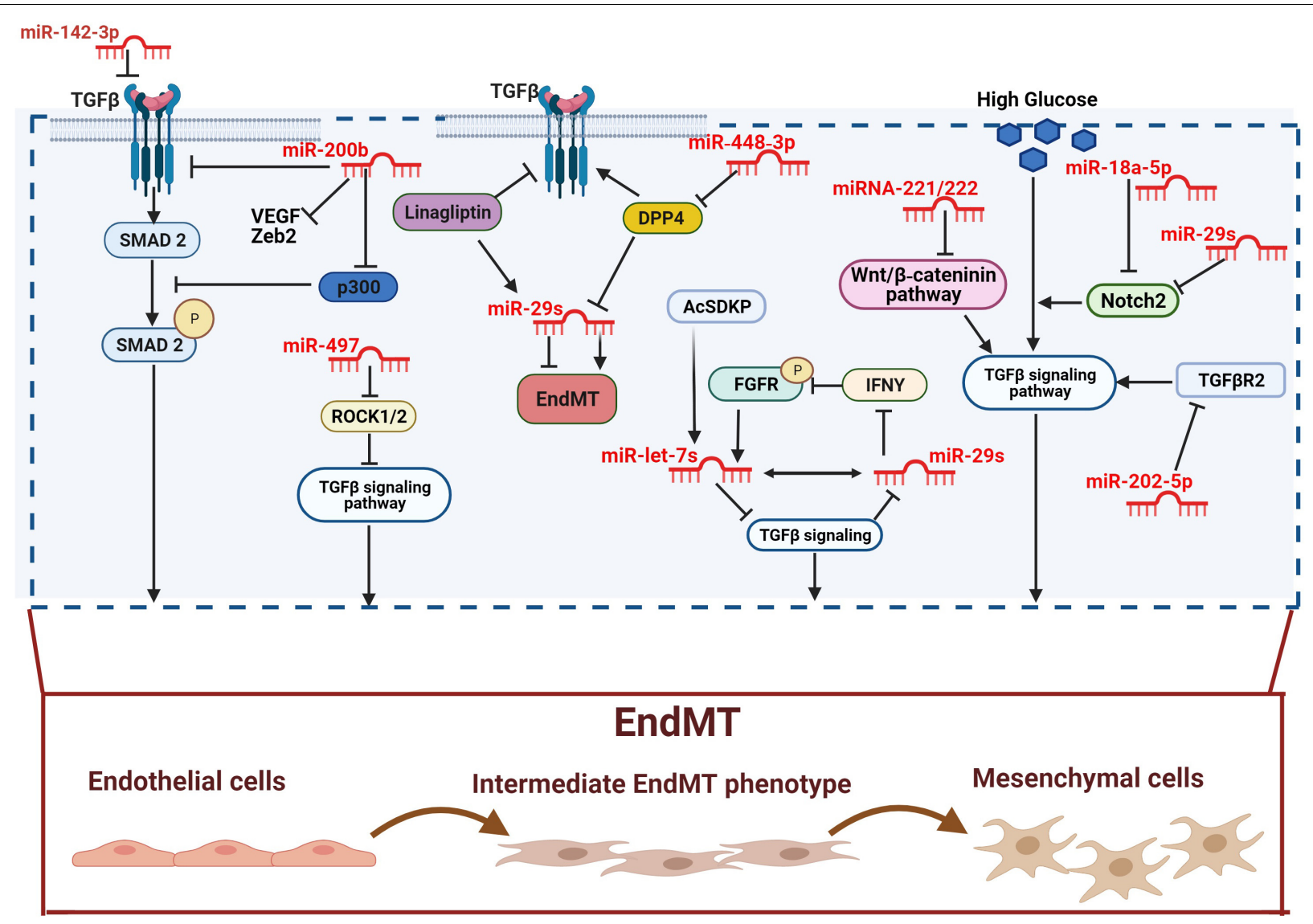

FIGURE 1 | Anti-fibrotic miRNAs in diabetic complications. miR-142-3p and miR-200b inhibit EndMT by inactivating the TGF- $\beta$-SMAD pathway. The antifibrotic activity of miR-200b is played by down-regulating the TGF- $\beta$ /SMAD pathway coactivator p300. miR-497 suppresses TGF- $\beta$-induced EndMT by ROCK1 and ROCK2 inactivation. The overexpression of DPP-4 is associated with the suppression of the miR-29s family anti-fibrotic activity. However, both linagliptin and AcSDKP suppresses EndMT by restoring miR-29 and miR-let-7s activities. Furthermore, miR-448-3p inhibits EndMT via DPP-4 suppression. AcSDKP upregulates the antifibrotic miR-let-7 which suppresses TGF $\beta$ R1 and TGF $\beta$ signaling. The block of TGF $\beta$ signaling results in up-regulation of miR-29 gene expression, which in turn causes FGFR1 phosphorylation. FGFR1 phosphorylation is critical for miR-let-7 production. miR-29 can also target the profibrotic IFNY blocking its inhibitory action toward FGFR1. The miR-29s family inhibits high glucose-induced EndMT by down-regulating Notch2, which is also suppressed by miR-18a-5p. However, DPP-4 inhibitor and AcSDKP suppresses EndMT by restoring of miR-29 and miR-let-7s activities. Furthermore, miR-448-3p inhibit EndMT via DPP-4 suppression. The miR-29s family inhibits high glucose-induced EndMT by the downregulation of Notch2 which is also suppressed by miR-18a-5p. High glucose-induced EndMT is also suppressed by miR-221/222 family, via the negative regulation of Wnt/ $\beta$-catenin, and by miR-202-5p via inhibition of TGF $\beta$ R2/TGF $\beta$ signaling pathway. Pro-fibrotic miRNAs are showed in dark, anti-fibrotic miRNAs in red.

TABLE 1 | Anti-fibrotic miRNAs in diabetic complications.

\section{Anti-fibrotic miRNAs in diabetic complications}

\begin{tabular}{|c|c|c|c|c|c|}
\hline miRNAs & DN & DR & Other & DCM & References \\
\hline miR-142-3p & & & & TGF $\beta-S M A D$ & Zhu et al., 2018 \\
\hline miR-200b miR-200b & & TGF $\beta 1-p 300$ & & TGF $\beta$-p300 & Cao et al., 2014; Feng et al., 2016 \\
\hline miR-497b & ROCK $1 / 2$ & & & & Liu et al., 2018 \\
\hline miR-221/222 miR-221/222 & & & & Wnt- $\beta /$ Catenin & Verjans et al., 2018; Wang et al., 2020 \\
\hline miR-448-3p & & & TGF $\beta$ signaling & & Guan et al., 2020 \\
\hline$m i R-18 a-5 p$ & & & & Notch2 & Geng and Guan, 2017 \\
\hline
\end{tabular}

Summarizes the references describing the anti-fibrotic miRNAs in diabetic complication.

$D N$, diabetic nephropathy; $D R$, diabetic retinopathy; DCM, diabetic cardiomyopathy. 


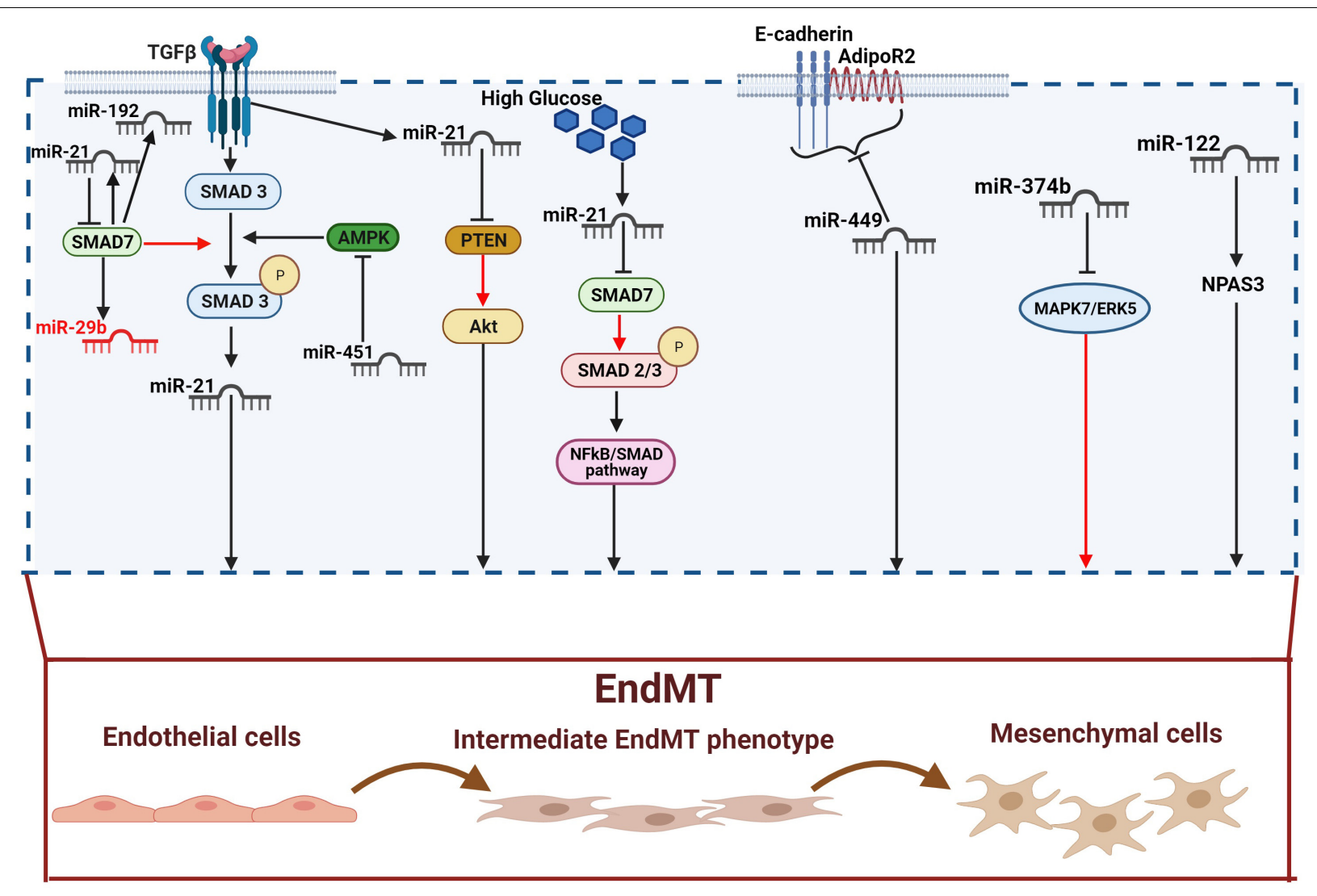

FIGURE 2 | Pro-fibrotic miRNAs in diabetic complications. TGF- $\beta$ increases miR-21 expression through Smad3 activation. miR-21 expression is also directly increased by TGF- $\beta$ and high glucose. miR-21 can in turn activates EndMT through releasing PTEN of Smad7 inhibition (red arrow). Indeed, both PTEN and SMAD7 are negative regulators of EndMT via the Akt and TGF- $\beta 1 /$ Smad3 signaling respectively. SMAD7 can also suppress fibrosis by down-regulating the pro-fibrotics miR-21 and miR-192, and up-regulating the anti-fibrotic miR-29b. miR451 triggers EndMT by blocking AMPK, an inhibitor of the TGF- $\beta /$ SMAD pathway. miR-449a induces EndMT by inhibiting AdipoR2 and E-cadherin interaction in the lipid rafts. miR-374b plays its profibrotic activity by releasing MAPK7/ERK5-mediated EndMT inhibition. Finally, miR-122 activates EndMT via the neuronal PAS domain protein 3 (NPAS3). Pro-fibrotic miRNAs are shown in dark, anti-fibrotic miRNAs in red.

TABLE 2 | Pro-fibrotic miRNAs in diabetic complications.

Pro-fibrotic miRNAs in diabetic complications

\begin{tabular}{|c|c|c|c|c|c|}
\hline miRNAs & DN & DR & AS & DCM & References \\
\hline miR-21 & PTEN/Akt & & & & Kumarswamy et al., 2012 \\
\hline miR-451 & & & & TGF $\beta$-SMAD & Liang et al., 2019 \\
\hline miR-449 & & & E-cadherin/AdipoR2 & & Jiang et al., 2019 \\
\hline miR-374b & & & MAPK7/ERK & & Vanchin et al., 2019 \\
\hline
\end{tabular}

Summarizes the references describing the pro-fibrotic miRNAs in diabetic complications.

$D N$, diabetic nephropathy; DR, diabetic retinopathy; DCM, diabetic cardiomyopathy; AS, atherosclerosis.

glycation end-products (AGEs) and Ang II, which in turn induce mitochondrial dysfunction in cardiomyocytes and ECs (Tan et al., 2002; Dikalov and Nazarewicz, 2013; Yan et al., 2014; Brunvand et al., 2017). Mitochondrial dysfunction, as well as the Ang II-induced NADPH oxidases stimulation, increases ROS production and oxidative stress (Dikalov and Nazarewicz, 2013; Siasos et al., 2018). Additionally, oxidative stress is also increased by lipid accumulation caused by an insulin resistance-induced cardiomyocytes metabolic shift. Indeed, the increased intake of fatty acid is not adequately metabolized by $\beta$-oxidation resulting in lipotoxicity (Boudina and Abel, 2010; Tan et al., 2020). Oxidative stress can in turn trigger endoplasmic reticulum (ER) stress, impairment of mitochondrial $\mathrm{Ca}^{2+}$ uptake, cardiomyocyte hypertrophy, ECs damage, microvascular dysfunction and the 
profibrotic responses by fibroblasts and inflammatory cells (Boudina and Abel, 2010; Tan et al., 2020). All these effects contribute to the accumulation of ECM, especially collagen type I and III, leading to myocardial fibrosis (Jia et al., 2018; Gollmer et al., 2019). The main signaling pathways underlying these pathophysiological events include TGF $\beta / S M A D, N F \kappa B / S M A D$, PKC, MAPK, Wnt/ $\beta$-catenin, Notch 2 and AcSDKP-FGFR1 signaling pathway (Nemir et al., 2014; $\mathrm{Hu}$ et al., 2018; $\mathrm{Ma}$ et al., 2018; Hortells et al., 2019; Li et al., 2020b; Yousefi et al., 2020). Most of these pathways lead to the development of cardiac fibrosis through the differentiation of fibroblasts into myofibroblasts as well as the endothelial-to-mesenchymal or epithelial-to-mesenchymal transition (Kovacic et al., 2012). Furthermore, increasing evidence suggests that miRNAs are the main players in the regulation of multiple pathways and cellular processes leading to cardiac fibrosis (Guo and Nair, 2017; Nandi and Mishra, 2018; Yousefi et al., 2020).

\section{miRNAs REGULATION OF DCM-ASSOCIATED EndMT}

The hyperglycemia-induced ECs damage and activation, resulting in vascular remodeling and EndMT, has been confirmed in myocardial fibrosis (Sharma et al., 2017). As suggested by experimental evidence, cardiac fibrogenesis involves the presence of a subset of EndMT-derived activated cardiac fibroblasts (Widyantoro et al., 2010; Sharma et al., 2017; Sánchez-Duffhues et al., 2018). Similarly, miRNAs are an important regulatory mechanism in cardiac fibrosis and heart failure (Wang Z. et al., 2016; Wang and Cai, 2017). In this context, miR-21, which has been widely described in pulmonary and renal fibrosis (Liu et al., 2016), plays an important role also in the pathogenesis of cardiac fibrosis and DCM (Adam et al., 2012; Guo and Nair, 2017; Yuan et al., 2017; Dai et al., 2018). A recent in vivo study confirmed the involvement of miR-21 in EndMT activation and myocardial fibrosis, showing that the hyperglycemia-induced up-regulation of miR-21 in diabetic mice is associated with the down-regulation of endothelial markers and the up-regulation of fibroblast markers (Li Q. et al., 2020). Moreover, similarly to the mechanism described in diabetic nephropathy (Zhong et al., 2011), miR-21 regulates EndMT through the NF-кB-SMAD signaling pathway by targeting SMAD7. The consequent SMAD7 inhibition increases SMAD2 and SMAD3 phosphorylation, resulting in EndMT activation (Li Q. et al., 2020). An additional mechanism, requiring the TGF$\beta /$ SMAD pathway, involves miR-142-3p, which has been shown to attenuate the hyperglycemia-induced EndMT in human aortic endothelial cells (HAECs) (Zhu et al., 2018). Indeed, miR-142-3p overexpression inhibits EndMT by inactivating both TGF- $\beta 1$ and the downstream target gene SMAD2. By contrast, TGF- $\beta 1$ overexpression significantly abolishes the inhibitory effects of miR-142-3p (Zhu et al., 2018). A negative regulation of glucoseinduced EndMT in the heart is also played by miR-200b (Feng et al., 2016). In a recent study, the expression of specific fibrotic markers, such as vascular endothelial growth factor (VEGF) (Yang et al., 2014), zinc finger E-box-binding homeobox (Zeb2)
(Jahan et al., 2018), and TGF- $\beta 1$ (Biernacka et al., 2011) was prevented in diabetic mice overexpressing miR-200b (Feng et al., 2016). Moreover, miR-200b overexpression also induces the down-regulation of p300, a transcription coactivator known to contribute to cardiac fibrosis and hypertrophy via TGF- $\beta / S M A D$ (Bugyei-Twum et al., 2014; Feng et al., 2016). Although the inhibitory role of the whole miR-200 family is well established, both in EMT (Korpal and Kang, 2008; Korpal et al., 2008) and EndMT (Feng et al., 2016; Zhang et al., 2017), unexpectedly a recent study shown that miR-200c-3p exerted the opposite effect, being able to promote EndMT and aortic graft remodeling both in vivo and in vitro (Chen et al., 2021). Finally, a further TGF$\beta /$ SMAD pathway-mediated regulatory mechanism involves miR-451 whose effects on EndMT are AMPK-dependent. Indeed, miR451 knockdown in diabetic mouse hearts suppresses EndMT through the activation of AMPK, which in turn inhibits the TGF- $\beta /$ SMAD pathway (Liang et al., 2019). As previously mentioned, in addition to TGF- $\beta /$ SMAD, other pathways underlie the pathophysiological events leading to cardiac fibrosis. One of them is the Wnt signaling pathway, known to promote fibroblast activation and proliferation (Tao et al., 2016). On the other hand, the anti-fibrotic role of miRNA-221/222 family has been confirmed, as their down-regulation was associated with heart failure (Verjans et al., 2018). The interplay between Wnt and miR-222 in EndMT regulation has been recently suggested (Wang et al., 2020); specifically, miR-222 is able to suppress the hyperglycemia-induced EndMT and inhibit cardiac fibrosis by negatively regulating the $\mathrm{Wnt} / \beta$-catenin pathway in diabetic mice (Wang et al., 2020). Lastly, a further protective effect versus EndMT is exerted through the notch pathway and involves miR-18a-5p (Geng and Guan, 2017). The role of the notch pathway in heart development and control of the balance between fibrotic and regenerative repair in the adult heart has been widely confirmed (Nemir et al., 2014). Moreover, Notch2 activation results essential for driving ECs differentiation (Noseda et al., 2004; Kovacic et al., 2019) in cardiovascular disease and for promoting EndMT independently or in association with TGF- $\beta /$ SMAD3 signaling (Fu et al., 2009; Chang et al., 2011). Notch2 is a target of miR-18a-5p which recently confirmed its antifibrotic role via the suppression of Notch2 and consequent inhibition of hyperglycemia-induced EndMT in human aortic valvular endothelial cells (HAVECs) (Geng and Guan, 2017). See figures and associated tables to overview of the signaling pathways involving both antifibrotic (Figure 1 and Table 1) and pro-fibrotic (Figure 2 and Table 2) miRNAs.

\section{DIABETIC RETINOPATHY}

Diabetic retinopathy (DR) is a common and severe microvascular complication of the eye that represents the leading cause of blindness in diabetes (Sabanayagam et al., 2016). The prevalence increases with disease progression and consequently with the exposure to the major risk factors, hyperglycemia and hypertension (Ding and Wong, 2012; Lee et al., 2015). Generally, a tight blood glucose control is cornerstone to reduce 
the risk of DR progression (Cheung and Wong, 2008). The condition is initially characterized by an asymptomatic stage, non-proliferative diabetic retinopathy (NPDR), that involves increased vascular permeability and capillary occlusion. Retinal neovascularization, by contrast, predominates in a later stage, proliferative diabetic retinopathy (PDR) (Lechner et al., 2017; Kusuhara et al., 2018), as consequence of hypoxia. However, as new vessels are relatively fragile, they tend to bleed into the macular region causing vision difficulties and, in the worst-case scenario, diabetic macular edema (DME), the main cause of blindness in DR (Wang and Lo, 2018). DME is described as a swelling of the macula due to fluid accumulation following breakdown of the blood-retinal barrier (BRB). This event can occur both in the PDR and in the NPDR stage (Das et al., 2015; Romero-Aroca et al., 2016). The BRB is composed of two distinct barriers: the outer $\mathrm{BRB}$, consisting of retinal pigment epithelium and the inner BRB, composed of endothelial cells regulating the transport across retinal capillaries. Besides, the BRB is established by tight cellular junctions, both in the inner and outer barrier, as well as by the scarcity of endocytic vesicles within cells, which further ensure the integrity of the BRB (Klaassen et al., 2013; Díaz-Coránguez et al., 2017). In addition, pericytes, specialized mural cells with a central role in angiogenesis, regulate and stabilize this tight structure through the Angiopoietin-1/Tie-2, platelet-derived growth factor (PDGF) and TGF- $\beta$ signaling pathways (Caporarello et al., 2019; Trost et al., 2019). BRB breakdown is a complex process involving different mechanisms; it can occur either in the inner BRB, the outer BRB, or both sites. The loss of integrity of the endothelial cell-cell junctions, the loss of pericytes and the thickening of the basement membrane are the major alterations observed in the inner BRB (Hammes et al., 2011; Das et al., 2015). Several studies have shown that hyperglycemia represents the main risk factor contributing to the pathogenesis of diabetic retinopathy (Engerman and Kern, 1986; Das et al., 2015; Eshaq et al., 2017). Furthermore, using a BRB model formed by retinal pericytes, astrocytes and endothelial cells, it has been recently reported that high glucose exposure elicits BRB breakdown, enhances $\mathrm{BRB}$ permeability and reduces the levels of junction proteins such as ZO-1 and VE-cadherin (Fresta et al., 2020). Besides, elevated ROS as well as pro-inflammatory mediators (IL-1 $\beta$, IL-6) and oxidative stress-related enzymes (iNOS, Nox2) have also been shown to be increased (Fresta et al., 2020). The major biochemical pathways involved in the BRB breakdown are the polyol pathway, the AGEs pathway, the PKC pathway and the hexosamine pathway. Oxidative stress and inflammation are responsible for the upregulation of growth factors and cytokines, such as VEGF, tumor necrosis factor (TNF), interleukins (ILs), and matrix metalloproteinases (MMPs), which contribute to the BRB breakdown and to the development of DME (Aiello et al., 1994; Brownlee, 2005; Gupta et al., 2013; Das et al., 2015). Studies have confirmed the role of the pro-angiogenic factor VEGF as main modulator of PDR and DME. VEGF is secreted by retinal pigmented epithelial cells, pericytes, and endothelial cells in response to hypoxia conditions caused by the obstruction and loss of retinal capillaries (Gupta et al., 2013; Romero-Aroca et al., 2016). VEGF, in addition to promoting neovascularization in $\mathrm{PDR}$, participates in the breakdown of the BRB via increasing permeability of retinal vessels (Ray et al., 2004). Indeed, high levels of VEGF increase the expression of the inflammatory intercellular adhesion molecule-1 (ICAM-1) which in turn facilitates the adhesion of leukocytes to the diabetic retinal vasculature, promoting capillary occlusion (Aiello et al., 1994; Joussen et al., 2002; Romero-Aroca et al., 2016).

\section{miRNAs REGULATION OF DR-ASSOCIATED EndMT}

Hyperglycemia-induced increased production of ECM and thickening of the vascular basement membrane is the hallmark of diabetic retinopathy (Roy et al., 2015). As previously mentioned, hyperglycemia promotes fibrosis progression through the generation of ECs-derived myofibroblasts, EndMT. This process has been shown to play an important role also in the pathogenesis of DR (Cao et al., 2014). Similar to other diabetic complications, TGF- $\beta$ is an important EndMT mediator, mainly through the activation of the SMAD signaling pathways (Van Geest et al., 2010; Cao et al., 2014; Pardali et al., 2017). Moreover, the transcriptional activator p300, already known for increasing the expression of ECM proteins (Kaur et al., 2006), and miR-200b have been described as key regulators of the TGF- $\beta$-mediated EndMT in diabetic mice (Cao et al., 2014). Although the specific mechanism played by miR-200b and p300 remains partially unknown, the anti-fibrotic activity of miR-200b, already described in other diabetic complications (McArthur et al., 2011; Feng et al., 2016), has also been confirmed in DR. Specifically, the EndMT observed in the retinas of wild-type diabetic mice was suppressed by the overexpression of miR-200b (Cao et al., 2014). As mentioned before, the outer BRB is composed of tight junctions of retina pigment epithelial cells (RPECs) which secrete various factors, nutrients and signaling molecules that influence the surrounding tissues (Campbell and Humphries, 2013; Liu and Liu, 2019). Chronic hyperglycemia alters RPECs functions contributing to the fluid accumulation in DME and the development of DR (Desjardins et al., 2016). Under stress conditions RPECs cells can release large amounts of exosomes, nanoscale vesicles that mediate many intercellular activities such as cell-to-cell communication, immune regulation, inflammatory response, extracellular matrix turnover and neovascularization (Klingeborn et al., 2017; Liu et al., 2020). A recent study confirmed the importance of the crosstalk between ECs and RPECs cells in the progression of fibrosis in patients with DR (Gu et al., 2020). Specifically, it was observed that hyperglycemia increased the ability of RPECs to release miR-202-5p-enriched exosomes. On the other hand, hyperglycemia induced EndMT through the TGF $\beta$ signaling pathway activation in ECs. However, when ECs were treated with RPECs-derived exosomes, the hyperglycemia-induced TGF $\beta$ signaling pathway activation was significantly counteracted as well as the increased proliferation and migration (Gu et al., 2020). In addition, miR-202-5p, by targeting specifically TGF $\beta$ R2, was responsible for the TGF $\beta$ signaling pathway inactivation and EndMT suppression 
(Gu et al., 2020). This study, in addition to providing additional evidence that hyperglycemia-induced EndMT involves the activation of TGF $\beta$ signaling, also showed that the release of miR-202-5p-enriched exosomes from RPE cells leads to the suppression of EndMT. The RPE cells-derived exosomes are therefore important mediators of the ECs-RPE cells crosstalk in the development of DR ( $\mathrm{Gu}$ et al., 2020). Additional miRNAs involved in EndMT regulation in DR include two members of the mi-RNA29 family, miR-29a and miR-29b, already described in fibrosis development associated with diabetic complications (He et al., 2013; Kanasaki et al., 2014; Zhang Y. et al., 2014; Srivastava et al., 2020a). The anti-fibrotic activity of miR$29 \mathrm{a} / \mathrm{b}$ has been recently confirmed also in DR where their overexpression suppressed the hyperglycemia-induced EndMT in human retinal microvascular endothelial cells (HRMECs) (Zhang et al., 2019). The inhibitory effect of miR-29a/b was exerted through the down-regulation of the transmembrane protein Notch2, known to activate morphological and functional changes of ECs as well as promote EndMT (Tian et al., 2017; Zhang et al., 2019). See figures and associated tables to overview of the signaling pathways involving both antifibrotic (Figure $\mathbf{1}$ and Table 1) and pro-fibrotic (Figure 2 and Table 2) miRNAs.

\section{ATHEROSCLEROSIS}

Atherosclerosis (AS) is characterized by plaque formation, secondary to the deposition of fats, cholesterol, and calcium, which lead to ischemia and its clinical manifestations, such as myocardial infarction and stroke (Lnsis, 2000). Although AS is classically associated with alterations of lipid metabolism and hypercholesterolemia (Wang H.H. et al., 2017), its pathogenesis is more complex and involves various factors. Endothelial dysfunction and inflammation are key steps in the sequence of events leading to AS (Davignon and Ganz, 2004; Hansson, 2009). The presence of mechanical stress, such as blood flow turbulence, can activate the endothelium, which responds by recruiting monocytes, adhesion molecules and pro-inflammatory cytokines. Monocytes, facilitated by adhesion molecules and cytokines, infiltrate the intima and can differentiate in macrophages which actively participate in lipid uptake through phagocytosis (Ilhan and Kalkanli, 2015). Diabetes and AS share several pathological mechanisms (La Sala et al., 2019b); indeed, the metabolic alterations that drive the development of diabetes are also involved in the pathogenesis of atherosclerosis (Federici and Lauro, 2005; Poznyak et al., 2020). In addition, both type 1 and type 2 diabetes can either induce atherosclerosis and accelerate its progression (Poznyak et al., 2020). In this context, a crucial role is played by the prolonged exposure to hyperglycemia and insulin resistance which are responsible for the increased atherosclerosis-related inflammation of the arterial wall (Reddy et al., 2010; Katakami, 2017). In addition to triggering the onset and progression of diabetes, insulin resistance also promotes dyslipidemia, hypertension and other metabolic abnormalities, important components of the pro-atherogenic milieu (Semenkovich, 2006;
Katakami, 2017). At the same time, an insufficient insulin signaling elicits an abnormal lipid metabolism and glucose transport and increase the production of glucose in the liver. Pancreatic $\beta$ cells respond to hyperglycemia by increasing insulin secretion; however, the continued stimulation of $\beta$ cells leads to their progressive functional failure and diabetes development (Cavaghan et al., 2000; Mangiafico et al., 2011). Prolonged exposure to hyperglycemia increases oxidative stress (Yu et al., 2011; Volpe et al., 2018), the primary activator of signaling pathways driving AS and diabetes progression (Vanessa Fiorentino et al., 2013; Yuan et al., 2019). Overproduction of ROS increases the formation of AGEs, modifications of proteins or lipids that become non-enzymatically glycated (Moreno-Viedma et al., 2016; Katakami, 2017). AGEs are involved in each step of atherosclerosis, being responsible for monocyte migration into the sub-endothelial space, release of cytokines by macrophages and stimulation of vasoconstriction (Katakami, 2017). Moreover, the binding of AGEs to the receptor RAGE activates TGF- $\beta$, ERK, JNK, p38, NF-kB, PKC and the polyol pathways as well as maintaining the chronic pro-inflammatory state of the arterial wall (Katakami, 2017; Yamagishi and Matsui, 2018).

\section{miRNAs REGULATION OF AS-ASSOCIATED EndMT}

As previously mentioned, endothelial dysfunction driven by oxidative stress plays a critical role in the development of AS. Persistent activation of ECs induces EndMT, which contributes to both the initiation and the progression of atherosclerosis (Chen et al., 2015; Evrard et al., 2017). Moreover, the extent of EndMT in the human plaque appears to be strongly correlated with the severity of the disease (Souilhol et al., 2018). A recent study showed the up-regulation of 17 miRNAs in atherosclerotic plaques; among them, miR-449a, already known for its role in lipid and cholesterol anabolism as well as inflammation (Zhang H. et al., 2014), was significantly higher compared with normal arteries (Jiang et al., 2019). The authors reported that miR449a induces EndMT and promotes the development of AS by targeting the interaction between adiponectin receptor 2 (AdipoR2) and E-cadherin in lipid rafts (Jiang et al., 2019). In this context, miR-449a has displayed a multilevel and complex regulatory mechanism by promoting proliferation and enhancing the migrating ability of ECs as well as their expression of atherosclerotic markers (Jiang et al., 2019). The ability to induce EndMT was confirmed by the reduced $E$-cadherin expression concurrently with the increased expression of $\alpha$-SMA and SMAD3 (Jiang et al., 2019). miR-449a pro-atherosclerotic properties are exerted by inhibition AdipoR2 and E-cadherin migration into the lipid raft fractions of ECs and consequent suppression E-cadherin-AdipoR2 of interaction. Additionally, the authors reported that blocking miR-449a protects diabetic mice from developing AS (Jiang et al., 2019). Similarly to miR-449a, miR-374b was reported to be up-regulated both in atheroprone regions from mice and pigs and in TGF- $\beta 1$-treated ECs (Vanchin et al., 2019). Additionally, the overexpression 
of miR-374b was associated with a reduction in endothelial markers (VE-Cadherin and eNOS), and a concomitant increase of mesenchymal markers (TAGLN and Calponin). Besides, miR$374 \mathrm{~b}$ was able to induce EndMT through the silencing of the Mitogen-Activated Protein Kinase 7 (MAPK7) also known as ERK5 (Vanchin et al., 2019). MAPK7 is an antagonist of EndMT and its signaling activity is generally lost in vessel areas that are undergoing pathological remodeling (NithianandarajahJones et al., 2014; Krenning et al., 2016). Similarly, MAPK7 signaling activity was lost in the sites of vascular remodeling, providing an additional confirmation of the inhibitory action of miR-374b. By contrast, the recovery of MAPK7 signaling abrogated the pathological effect of miR-374b (Vanchin et al., 2019). miR-122, another miRNA recently reported as EndMT mediator in AS, has been shown to be up-regulated both in the aortic intima of diabetic mice and in the cellular EndMT model (Wu et al., 2021). The regulatory action of miR-122 is mediated by the neuronal PAS domain protein 3 (NPAS3). Indeed, inhibition of miR-122 prevented atherosclerosis and regulated NPAS3-mediated EndMT (Wu et al., 2021). miR122 might therefore represent a druggable target in preventing EndMT-associated atherosclerosis. See figures and associated tables to overview of the signaling pathways involving both antifibrotic (Figure $\mathbf{1}$ and Table 1) and pro-fibrotic (Figure 2 and Table 2) miRNAs.

\section{LONG NON-CODING RNAS REGULATION IN DIABETES-ASSOCIATED EndMT}

Besides miRNAs, small RNAs also include long non-coding RNAs (lncRNAs) and circular RNAs (circRNAs) which are emerging as key regulators implicated in a significant number of biological processes (Qu et al., 2017; Statello et al., 2020). Unlike linear RNAs, circRNAs form a covalently closed continuous loop, without $5^{\prime}$ or $3^{\prime}$ ends (Qu et al., 2015). IncRNAs are instead linear RNAs, with a nucleotide length $>200$, that can affect gene transcription both at the epigenetic, transcriptional and post-transcriptional level (Dykes and Emanueli, 2017; Wang C. et al., 2017). Thus, IncRNAs can differently interact with mRNAs, proteins, and DNA elements; moreover, the binding of transcriptional factors to the lncRNA promoter's target sites can regulate their expression (Taft et al., 2010). IncRNAs are also precursors of many types of miRNAs, although more frequently they overlap both physically and functionally with the latter. Moreover, IncRNAs compete with miRNAs for the binding to the same target genes and can trigger miRNAs degradation (Taft et al., 2010; Chen et al., 2018). Hence, lncRNAs are involved in a variety of human diseases where they appear differentially expressed or genetically perturbed (Harries, 2012; Shi et al., 2013). In this context, most of the knowledge pertaining to lncRNAs is derived from cancer however there is increasing evidence of their involvement in other conditions, such as Alzheimer's disease, diabetes, cardiac complications (DiStefano, 2018; Greco et al., 2018; Leung and Natarajan, 2018) and fibrosis (He Z. et al., 2020; Li et al., 2020a; Lin J. et al., 2020). One important function of lncRNAs is their role as a molecular sponge to certain miRNAs, hindering their expression (Biswas et al., 2018). This mechanism has been confirmed in diabetic kidney fibrosis, where the down-regulation of the anti-fibrotic miR-29 was associated with lncRNA H19 up-regulation, whereas its knockdown restored miR-29 activity and significantly inhibited TGF- $\beta 2$-induced EndMT in diabetic mice (Shi et al., 2020). However, the role of H19 in diabetesassociated EndMT remains unclear; indeed, H19 overexpression prevented glucose-induced EndMT by reducing the TGF- $\beta 1$ levels in DR (Thomas et al., 2019). Further studies are required to clarify the role of $\mathrm{H} 19$ in regulating EndMT in diabetic conditions. Another lncRNA involved in DR is the maternally expressed gene 3 (MEG3) which showed an inhibitory effect on hyperglycemia-induced EndMT. MEG3 resulted indeed able to suppress EndMT both in vivo and in vitro by inhibiting the PI3K/AKT/mTOR signaling pathway (He Y. et al., 2020). On the other hand, MEG3 methylation mediated by DNA methyltransferase 1 (DNMT1) attenuated MEG3 expression and consequently accelerated EndMT (He Y. et al., 2020). This finding clarifies the role of MEG3 in EndMT and provide additional confirmation that increased levels of DNA methylation represent a potential risk factor for the development of DR (Maghbooli et al., 2015). As previously reported, oxidized low density lipoproteins (ox-LDL), being able to trigger plaque formation and EndMT, are key players in AS development (Su et al., 2018). A recent study reported that miR-30c-5p and LINC00657, also known as non-coding RNA activated by DNA damage (NORAD), are both involved in ox-LDLinduced EndMT but with opposite effects (Wu et al., 2020). miR-30c-5p inhibited ox-LDL-induced EndMT via activation of the Wnt7b/ $\beta$-catenin pathway whereas LINC00657, acting as sponge of miR-30c-5p, suppressed the EndMT inhibition (Wu et al., 2020). Indeed, the expression level of LINC00657 resulted elevated both in sera from AS patients and in ox-LDL-stimulated ECs (Wu et al., 2020).

\section{POTENTIAL ROS-ENdMT-SMALL RNAS INTERPLAY IN DIABETES-ASSOCIATED FIBROTIC CONDITIONS}

Oxidative stress is a key player in the diabetic complications' pathophysiology described in this review. Hyperglycemia is not only the main factor responsible for the increase in ROS but also favors the increase of inflammatory mediators, which ultimately leads to vascular dysfunction (Luc et al., 2019). Both genetic and epigenetic factors can regulate the development and exacerbation of oxidative stress; in this context, different studies have highlighted the key role played by miRNAs (Grieco et al., 2019). Indeed, hyperglycemia can alter miRNAs expression, which in turn contributes to the development of endothelium dysfunction and diabetic vascular disease (Luc et al., 2019). Besides, in diabetic complications the molecular mechanisms and signaling pathways triggered by oxidative stress appear similar to those involved in miRNAs regulation (Grieco et al., 2019; Qadir et al., 2019). Finally, hyperglycemia-induced oxidative stress can affect the expression 
of specific miRNAs, which in turn can exacerbate oxidative stress, in addition to regulating the fibrotic process through the mechanisms summarized in this review (Grieco et al., 2019; Qadir et al., 2019). On the other hand, oxidative stress is emerging as a key trigger of EndMT (Montorfano et al., 2014; Thuan et al., 2018). Therefore, although a direct oxidative stress-small RNAs-EndMT link has not been demonstrated in diabetes yet, a substantial body of evidence supports this interplay. For example, an indirect proof of a ROS-miR-21-EndMT link has been reported with kallistatin, an endogenous protein with beneficial effects on EndMT-associated fibrosis (Guo et al., 2015). Kallistatin treatment blocked TGF$\beta$-induced EndMT, NADPH oxidase-dependent ROS formation and the expression of the pro-fibrotic miR-21, confirming the role of both miR-21 and ROS as major mediators of EndMT (Guo et al., 2015). Many studies indicated a direct link between mi-R21 and oxidative stress in diabetic subjects, where ROS generation has been suggested as a downstream effect of miR-21 overexpression (La Sala et al., 2019a). The prooxidant effect of miR-21 is exerted through the suppression of genes which usually limit oxidative damage such as KRIT1 (Krev/Rap1 Interaction Trapped-1), Nuclear Factor erythroid Related Factor 2 (NRF2), and MnSOD2 (Manganese-dependent Superoxide Dismutase2). By contrast, inhibition of miR-21 decreases ROS levels (La Sala et al., 2018; Grieco et al., 2019). A relationship between up-regulation of miR-21 and increased ROS levels has also been shown during the development of diabetic cardiac dysfunctions (Yildirim et al., 2013). The miR-200 family, the anti-fibrotic activity of which has been described both in diabetic nephropathy and retinopathy, has also been shown to be associated with a decrease in oxidative stress in diabetes; specifically, the antioxidant effect of miR200 is exerted by silencing the O-GlcNAc transferase, also known as OGT, whose enzymatic activity is associated with diabetic complications and endothelial inflammation (Qadir et al., 2019). Another proof of the oxidative stress-small RNAsEndMT interconnection comes from a study investigating the activity of miR-451 (Ruknarong et al., 2021). The latter, previously described for its ability to induce EndMT in diabetic mouse heart (Liang et al., 2019), has been recently reported to be up-regulated in diabetic subjects with high oxidative stress. The association between miR-451 and oxidative stress has been further confirmed with the use of the antioxidant Vitamin C; indeed, Vitamin C administration in diabetic subjects decreased both the expression of miR-451 and ROS levels (Ruknarong et al., 2021). Finally, an interplay being the basis of mitochondrial functions in kidney ECs involves the miR-let-7 family, (FGF)/FGFR1 signaling pathway and SIRT3 (Srivastava et al., 2020c). The integrity of the FGFR1-miR-let7 axis, on which depends the modulation of SIRT3, is crucial for maintaining the mitochondrial functionality (Srivastava et al., 2020c). SIRT3, for its part, controls mitochondrial redox homeostasis by modulation of ROS levels (Jing et al., 2011; Bause and Haigis, 2013) mainly via activation of the antioxidant enzyme superoxide-dismutase 2 (Qiu et al., 2010). On the contrary, the loss of the FGFR1-miR-let-7axis impairs SIRT3 and miR-29 levels with consequent disruption of mitochondrial integrity and activation of pro-mesenchymal signaling (Wnt signaling, BMP, Notch, TGF- $\beta$ signaling) promoting EndMT (Srivastava et al., 2020c).

\section{CONCLUSION AND FUTURE DIRECTIONS}

This review has highlighted the key role of EndMT in the fibrotic process occurring in the development of the major diabetic complications. Environmental factors (high glucose, hypoxia, oxidative stress, pro-inflammatory cytokines) are important determinants of EndMT induction through the activation of specific signaling pathways, such as TGF- $\beta$, Notch, Wnt, and the modulation of the expression of microRNAs. The evidence reviewed in this article indicates that some microRNAs, e.g., miR-29, miR-200, and miR-Let7, have anti-fibrotic effects and inhibit EndMT whereas others, e.g., miR-21 and miR122, possess pro-fibrotic properties and promote EndMT. The anti-fibrotic activity of some microRNAs appears univocal not only within diabetic complications but also in other pathological conditions. For instance, miR-29a/b and miR-200b have been shown to inhibit fibrosis in pulmonary fibrosis (Yang et al., 2012; Cushing et al., 2015), systemic sclerosis (Harmanci et al., 2017) as well as in DCM, DN, and DR (Cao et al., 2014; Kanasaki et al., 2014; Feng et al., 2016; Zhang et al., 2019). Similarly, miR-21 is generally up-regulated in different fibrotic diseases (Huang et al., 2015; Liu et al., 2016) as well as in diabetic complications such as DN, DR, and DCM (Srivastava et al., 2013; Chen et al., 2017; Li Q. et al., 2020). Moreover, since the expression levels of miR21 in the plasma of diabetic patients were correlated with disease progression, miR-21 might be used as a marker of diabetes severity (Jiang et al., 2017). On the other hand, the function of other microRNAs is only partially established in in vitro models or in specific pathological conditions. Further, for some miRNAs the evidence is still controversial, such as the case of the IncRNA H19 which showed pro-fibrotic activity in DN (Shi et al., 2020) and an opposite effect in DR (Thomas et al., 2019). Additionally, since the markers for EndMT used in individual studies are often different, a complete understanding of the regulatory mechanisms played by miRNAs, or an exact comparison between them, is currently challenging. In this regard, future directions in the study of diabetic complications should involve (a) a thorough characterization of the mechanisms involved in the ROS-EndMT-small RNAs interplay and its relationship with the onset and severity of specific complications, (b) the conduct of epidemiological studies investigating the association between specific miRNAs and lncRNAs and metabolic control, surrogate markers of organ damage, and morbidity and mortality in patients with diabetes, and (c) the effects of specific pharmacological and non-pharmacological interventions targeting EndMT on the risk and progression of diabetic complications. Such studies might contribute to the identification of new diagnostic and therapeutic strategies to prevent or limit the structural and functional damage that leads to organ and system failure in diabetes. 


\section{AUTHOR CONTRIBUTIONS}

RG, YMAA, and GP: conceptualization. GKN, AAM, and GP: resources. RG and YMAA: writing the original manuscript draft. RG, YMAA, HA, SA, LP, GKN, AAM, and GP: review and editing the different manuscript versions. AAM and GP: final editing and supervision. GP: submission. All authors: read and agreed to the published version of the manuscript.

\section{REFERENCES}

Adam, O., Löhfelm, B., Thum, T., Gupta, S. K., Puhl, S.-L., Schäfers, H.-J., et al. (2012). Role of miR-21 in the pathogenesis of atrial fibrosis. Basic Res. Cardiol. 107:278.

Aghadavoud, E., Nasri, H., and Amiri, M. (2017). Molecular signaling pathways of diabetic kidney disease; new concepts. J. Prevent. Epidemiol. 2, e09-e.

Aiello, L. P., Avery, R. L., Arrigg, P. G., Keyt, B. A., Jampel, H. D., Shah, S. T., et al. (1994). Vascular endothelial growth factor in ocular fluid of patients with diabetic retinopathy and other retinal disorders. N. Engl. J. Med. 331, 1480-1487. doi: 10.1056/nejm199412013312203

Anand, S., and Tamura, M. K. (2012). Combining angiotensin receptor blockers with ACE inhibitors in elderly patients. Am. J. Kidney Dis. Offic. J. Natl. Kidney Foundat. 59:11. doi: 10.1053/j.ajkd.2011.09.002

Badal, S. S., and Danesh, F. R. (2014). New insights into molecular mechanisms of diabetic kidney disease. Am. J. Kidney Dis. 63, S63-S83.

Bakker, W., Eringa, E. C., Sipkema, P., and van Hinsbergh, V. W. (2009). Endothelial dysfunction and diabetes: roles of hyperglycemia, impaired insulin signaling and obesity. Cell Tissue Res. 335, 165-189. doi: 10.1007/s00441-0080685-6

Ban, C. R., and Twigg, S. M. (2008). Fibrosis in diabetes complications: pathogenic mechanisms and circulating and urinary markers. Vascul. Health Risk Manage. 4:575. doi: 10.2147/vhrm.s1991

Batlle, D., Soler, M. J., and Ye, M. (2010). ACE2 and diabetes: ACE of ACEs? Diabetes 59, 2994-2996. doi: 10.2337/db10-1205

Bause, A. S., and Haigis, M. C. (2013). SIRT3 regulation of mitochondrial oxidative stress. Exp. Gerontol. 48, 634-639. doi: 10.1016/j.exger.2012.08.007

Benigni, A., Cassis, P., and Remuzzi, G. (2010). Angiotensin II revisited: new roles in inflammation, immunology and aging. EMBO Mol. Med. 2, 247-257. doi: 10.1002/emmm.201000080

Biernacka, A., Dobaczewski, M., and Frangogiannis, N. G. (2011). TGF- $\beta$ signaling in fibrosis. Growth Fact. 29, 196-202.

Biswas, S., Thomas, A. A., and Chakrabarti, S. (2018). LncRNAs: proverbial genomic "junk" or key epigenetic regulators during cardiac fibrosis in diabetes? Front. Cardiovasc. Med. 5:28. doi: 10.3389/fcvm.2018.00028

Bochaton-Piallat, M.-L., Gabbiani, G., and Hinz, B. (2016). The myofibroblast in wound healing and fibrosis: answered and unanswered questions. F1000Research 5, F1000FacultyRev-752.

Boudina, S., and Abel, E. D. (2010). Diabetic cardiomyopathy, causes and effects. Rev. Endocr. Metab. Disord. 11, 31-39. doi: 10.1007/s11154-010-9131-7

Brosius, F. C. (2008). New insights into the mechanisms of fibrosis and sclerosis in diabetic nephropathy. Rev. Endocr. Metab. Disord. 9, 245-254.

Brownlee, M. (2005). The pathobiology of diabetic complications: a unifying mechanism. Diabetes 54, 1615-1625. doi: 10.2337/diabetes.54.6.1615

Brunvand, L., Heier, M., Brunborg, C., Hanssen, K. F., Fugelseth, D., Stensaeth, K. H., et al. (2017). Advanced glycation end products in children with type 1 diabetes and early reduced diastolic heart function. BMC Cardiovasc. Disord. 17:1-6. doi: 10.1186/s12872-017-0551-0

Bugyei-Twum, A., Advani, A., Advani, S. L., Zhang, Y., Thai, K., Kelly, D. J., et al. (2014). High glucose induces Smad activation via the transcriptional coregulator p300 and contributes to cardiac fibrosis and hypertrophy. Cardiovasc. Diabetol. 13, 1-12.

Cabili, M. N., Trapnell, C., Goff, L., Koziol, M., Tazon-Vega, B., Regev, A., et al. (2011). Integrative annotation of human large intergenic noncoding RNAs reveals global properties and specific subclasses. Genes Dev. 25, 1915-1927. doi: $10.1101 /$ gad.17446611

\section{FUNDING}

This work has been made possible thanks to grants from the University of Sharjah (Seed 2001050151) to GP; (collaborative 2101050160) to GP and AAM; Qatar University (IRCC-2019-007) to GKN and GP; and (fondo UNISS di Ateneo per la Ricerca 2020) to GP.

Calle, P., and Hotter, G. (2020). Macrophage phenotype and fibrosis in diabetic nephropathy. Int. J. Mol. Sci. 21:2806. doi: 10.3390/ijms21082806

Campbell, M., and Humphries, P. (2013). The blood-retina barrier. Biology and Regulation of Blood-Tissue Barriers. Berlin: Springer, 70-84.

Cao, Q., Chen, X. M., Huang, C., and Pollock, C. A. (2019). MicroRNA as novel biomarkers and therapeutic targets in diabetic kidney disease: an update. FASEB BioAdv. 1, 375-388. doi: 10.1096/fba.2018-00064

Cao, Y., Feng, B., Chen, S., Chu, Y., and Chakrabarti, S. (2014). Mechanisms of endothelial to mesenchymal transition in the retina in diabetes. Investigat. Ophthalmol. Vis. Sci. 55, 7321-7331. doi: 10.1167/iovs.14-15167

Caporarello, N., D’Angeli, F., Cambria, M. T., Candido, S., Giallongo, C., Salmeri, M., et al. (2019). Pericytes in microvessels: from "mural" function to brain and retina regeneration. Int. J. Mol. Sci. 20:6351. doi: 10.3390/ijms20246351

Catalanotto, C., Cogoni, C., and Zardo, G. (2016). MicroRNA in control of gene expression: an overview of nuclear functions. Int. J. Mol. Sci. 17:1712. doi: 10.3390/ijms 17101712

Cavaghan, M. K., Ehrmann, D. A., and Polonsky, K. S. (2000). Interactions between insulin resistance and insulin secretion in the development of glucose intolerance. J. Clin. Investig. 106, 329-333. doi: 10.1172/jci10761

Chang, A. C., Fu, Y., Garside, V. C., Niessen, K., Chang, L., Fuller, M., et al. (2011). Notch initiates the endothelial-to-mesenchymal transition in the atrioventricular canal through autocrine activation of soluble guanylyl cyclase. Dev. Cell 21, 288-300. doi: 10.1016/j.devcel.2011.06.022

Chen, D., Zhang, C., Chen, J., Yang, M., Afzal, T. A., An, W., et al. (2021). miRNA$200 \mathrm{c}-3 \mathrm{p}$ promotes endothelial to mesenchymal transition and neointimal hyperplasia in artery bypass grafts. J. Pathol. 253, 209-224. doi: 10.1002/path. 5574

Chen, F., Yu, Y., Haigh, S., Johnson, J., Lucas, R., Stepp, D. W., et al. (2014). Regulation of NADPH oxidase 5 by protein kinase $\mathrm{C}$ isoforms. PLoS One 9:e88405. doi: 10.1371/journal.pone.0088405

Chen, P.-Y., Qin, L., Baeyens, N., Li, G., Afolabi, T., Budatha, M., et al. (2015). Endothelial-to-mesenchymal transition drives atherosclerosis progression. J. Clin. Investig. 125, 4514-4528. doi: 10.1172/jci82719

Chen, P.-Y., Qin, L., Barnes, C., Charisse, K., Yi, T., Zhang, X., et al. (2012). FGF regulates TGF- $\beta$ signaling and endothelial-to-mesenchymal transition via control of let-7 miRNA expression. Cell Rep. 2, 1684-1696. doi: 10.1016/j.celrep. 2012.10.021

Chen, Q., Qiu, F., Zhou, K., Matlock, H. G., Takahashi, Y., Rajala, R. V., et al. (2017). Pathogenic role of microRNA-21 in diabetic retinopathy through downregulation of PPAR $\alpha$. Diabetes 66, 1671-1682. doi: 10.2337/db16-1246

Chen, X., Sun, Y., Cai, R., Wang, G., Shu, X., and Pang, W. (2018). Long noncoding RNA: multiple players in gene expression. BMB Rep. 51:280. doi: 10.5483/ bmbrep.2018.51.6.025

Cheung, N., and Wong, T. Y. (2008). Diabetic retinopathy and systemic vascular complications. Prog. Retinal Eye Res. 27, 161-176. doi: 10.1016/j.preteyeres. 2007.12.001

Cho, J. G., Lee, A., Chang, W., Lee, M.-S., and Kim, J. (2018). Endothelial to mesenchymal transition represents a key link in the interaction between inflammation and endothelial dysfunction. Front. Immunol. 9:294. doi: 10.3389/ fimmu.2018.00294

Chung, A. C., Dong, Y., Yang, W., Zhong, X., Li, R., and Lan, H. Y. (2013). Smad7 suppresses renal fibrosis via altering expression of TGF- $\beta$ / Smad3-regulated microRNAs. Mol. Therap. 21, 388-398. doi: 10.1038/mt.20 12.251

Curci, C., Castellano, G., Stasi, A., Divella, C., Loverre, A., Gigante, M., et al. (2014). Endothelial-to-mesenchymal transition and renal fibrosis in 
ischaemia/reperfusion injury are mediated by complement anaphylatoxins and Akt pathway. Nephrol. Dial. Transplant. 29, 799-808. doi: 10.1093/ndt/ gft516

Cushing, L., Kuang, P., and Lü, J. (2015). The role of miR-29 in pulmonary fibrosis. Biochem. Cell Biol. 93, 109-118. doi: 10.1139/bcb-2014-0095

Dai, B., Li, H., Fan, J., Zhao, Y., Yin, Z., Nie, X., et al. (2018). MiR-21 protected against diabetic cardiomyopathy induced diastolic dysfunction by targeting gelsolin. Cardiovasc. Diabetol. 17, 1-17.

Das, A., McGuire, P. G., and Rangasamy, S. (2015). Diabetic macular edema: pathophysiology and novel therapeutic targets. Ophthalmology 122, 1375-1394. doi: 10.1016/j.ophtha.2015.03.024

Davignon, J., and Ganz, P. (2004). Role of endothelial dysfunction in atherosclerosis. Circulation 109, III-27-III-32.

Deacon, C. F. (2019). Physiology and pharmacology of DPP-4 in glucose homeostasis and the treatment of type 2 diabetes. Front. Endocrinol. 10:80. doi: 10.3389/fendo.2019.00080

Dejana, E., Hirschi, K. K., and Simons, M. (2017). The molecular basis of endothelial cell plasticity. Nat. Communicat. 8, 1-11. doi: 10.1007/978-159259-253-1_1

Deshpande, A. D., Harris-Hayes, M., and Schootman, M. (2008). Epidemiology of diabetes and diabetes-related complications. Phys. Therap. 88, 1254-1264. doi: $10.2522 / \mathrm{ptj} .20080020$

Desjardins, D. M., Yates, P. W., Dahrouj, M., Liu, Y., Crosson, C. E., and Ablonczy, Z. (2016). Progressive early breakdown of retinal pigment epithelium function in hyperglycemic rats. Investig. Ophthalmol. Vis. Sci. 57, 2706-2713. doi: 10. 1167/iovs.15-18397

Díaz-Coránguez, M., Ramos, C., and Antonetti, D. A. (2017). The inner bloodretinal barrier: Cellular basis and development. Vis. Res. 139, 123-137. doi: 10.1016/j.visres.2017.05.009

Dikalov, S. I., and Nazarewicz, R. R. (2013). Angiotensin II-induced production of mitochondrial reactive oxygen species: potential mechanisms and relevance for cardiovascular disease. Antioxid. Redox Signal. 19, 1085-1094. doi: 10.1089/ars. 2012.4604

Ding, J., and Wong, T. Y. (2012). Current epidemiology of diabetic retinopathy and diabetic macular edema. Curr. Diab. Rep. 12, 346-354. doi: 10.1007/s11892012-0283-6

DiStefano, J. K. (2018). The emerging role of long noncoding RNAs in human disease. Dis. Gene Identificat. 1706, 91-110. doi: 10.1007/978-1-4939-7471-9_6

Dykes, I. M., and Emanueli, C. (2017). Transcriptional and post-transcriptional gene regulation by long non-coding RNA. Genomics Proteom. Bioinformat. 15, 177-186. doi: 10.1016/j.gpb.2016.12.005

Engerman, R. L., and Kern, T. S. (1986). Hyperglycemia as a cause of diabetic retinopathy. Metabolism 35, 20-23. doi: 10.1016/0026-0495(86) 90182-4

Eshaq, R. S., Aldalati, A. M., Alexander, J. S., and Harris, N. R. (2017). Diabetic retinopathy: breaking the barrier. Pathophysiology 24, 229-241. doi: 10.1016/j. pathophys.2017.07.001

Evcimen, N. D., and King, G. L. (2007). The role of protein kinase C activation and the vascular complications of diabetes. Pharmacol. Res. 55, 498-510. doi: 10.1016/j.phrs.2007.04.016

Evrard, S. M., Lecce, L., Michelis, K. C., Nomura-Kitabayashi, A., Pandey, G., Purushothaman, K.-R., et al. (2016). Endothelial to mesenchymal transition is common in atherosclerotic lesions and is associated with plaque instability. Nat. Commun. 7, 1-16.

Evrard, S. M., Lecce, L., Michelis, K. C., Nomura-Kitabayashi, A., Pandey, G., Purushothaman, K.-R., et al. (2017). Corrigendum: endothelial to mesenchymal transition is common in atherosclerotic lesions and is associated with plaque instability. Nat. Communicat. 8:14710.

Fan, B., Chopp, M., Zhang, Z. G., and Liu, X. S. (2020). Emerging roles of microRNAs as biomarkers and therapeutic targets for diabetic neuropathy. Front. Neurol. 11:558758. doi: 10.3389/fneur.2020.558758

Federici, M., and Lauro, R. (2005). Diabetes and atherosclerosis-running on a common Road. Aliment. Pharmacol. Therapeut. 22, 11-15. doi: 10.1111/j.13652036.2005.02617.x

Feng, B., Cao, Y., Chen, S., Chu, X., Chu, Y., and Chakrabarti, S. (2016). miR-200b mediates endothelial-to-mesenchymal transition in diabetic cardiomyopathy. Diabetes 65, 768-779. doi: 10.2337/db15-1033
Feng, S.-D., Yang, J.-H., Yao, C. H., Yang, S.-S., Zhu, Z.-M., Wu, D., et al. (2017). Potential regulatory mechanisms of $\operatorname{lncRNA}$ in diabetes and its complications. Biochem. Cell Biol. 95, 361-367. doi: 10.1139/bcb-2016-0110

Ferreira, F. U., Souza, L. E. B., Thomé, C. H., Pinto, M. T., Origassa, C., Salustiano, S., et al. (2019). Endothelial cells tissue-specific origins affects their responsiveness to TGF- $\beta 2$ during endothelial-to-mesenchymal transition. Int. J. Mol. Sci. 20:458. doi: 10.3390/ijms20030458

Frantz, C., Stewart, K. M., and Weaver, V. M. (2010). The extracellular matrix at a glance. J. Cell Sci. 123, 4195-4200. doi: 10.1242/jcs.023820

Fresta, C. G., Fidilio, A., Caruso, G., Caraci, F., Giblin, F. J., Leggio, G. M., et al. (2020). A new human blood-retinal barrier model based on endothelial cells, pericytes, and astrocytes. Int. J. Mol. Sci. 21:1636. doi: 10.3390/ijms21051636

Fu, Y., Chang, A., Chang, L., Niessen, K., Eapen, S., Setiadi, A., et al. (2009). Differential regulation of transforming growth factor $\beta$ signaling pathways by Notch in human endothelial cells. J. Biol. Chem. 284, 19452-19462. doi: 10. $1074 /$ jbc.m109.011833

Geng, H., and Guan, J. (2017). MiR-18a-5p inhibits endothelial-mesenchymal transition and cardiac fibrosis through the Notch2 pathway. Biochem. Biophys. Res. Commun. 491, 329-336. doi: 10.1016/j.bbrc.2017.07.101

Ghosh, A. K., Nagpal, V., Covington, J. W., Michaels, M. A., and Vaughan, D. E. (2012). Molecular basis of cardiac endothelial-to-mesenchymal transition (EndMT): differential expression of microRNAs during EndMT. Cell. Signal. 24, 1031-1036. doi: 10.1016/j.cellsig.2011.12.024

Giacchetti, G., Sechi, L. A., Rilli, S., and Carey, R. M. (2005). The reninangiotensin-aldosterone system, glucose metabolism and diabetes. Trends Endocrinol. Metabol. 16, 120-126. doi: 10.1016/j.tem.2005.02.003

Giordo, R., Nasrallah, G. K., Posadino, A. M., Galimi, F., Capobianco, G., Eid, A. H., et al. (2021). Resveratrol-Elicited PKC Inhibition Counteracts NOX-Mediated Endothelial to Mesenchymal Transition in Human Retinal Endothelial Cells Exposed to High Glucose. Antioxidants 10:224. doi: 10.3390/antiox100 20224

Glover, E. K., Jordan, N., Sheerin, N. S., and Ali, S. (2019). Regulation of endothelial-to-mesenchymal transition by microRNAs in chronic allograft dysfunction. Transplantation 103:e64. doi: 10.1097/tp.0000000000002589

Goldin, A., Beckman, J. A., Schmidt, A. M., and Creager, M. A. (2006). Advanced glycation end products: sparking the development of diabetic vascular injury. Circulation 114, 597-605. doi: 10.1161/circulationaha.106.621854

Gollmer, J., Zirlik, A., and Bugger, H. (2019). Established and emerging mechanisms of diabetic cardiomyopathy. J. Lipid Atheroscler. 8:26. doi: 10. 12997/jla.2019.8.1.26

Greco, S., Salgado Somoza, A., Devaux, Y., and Martelli, F. (2018). Long noncoding RNAs and cardiac disease. Antioxid. Redox Signal. 29, 880-901.

Grieco, G. E., Brusco, N., Licata, G., Nigi, L., Formichi, C., Dotta, F., et al. (2019). Targeting microRNAs as a therapeutic strategy to reduce oxidative stress in diabetes. Int. J. Mol. Sci. 20:6358. doi: 10.3390/ijms20246358

Gross, J. L., De Azevedo, M. J., Silveiro, S. P., Canani, L. H., Caramori, M. L., and Zelmanovitz, T. (2005). Diabetic nephropathy: diagnosis, prevention, and treatment. Diab. Care 28, 164-176.

Gu, S., Liu, Y., Zou, J., Wang, W., Wei, T., Wang, X., et al. (2020). Retinal pigment epithelial cells secrete miR-202-5p-containing exosomes to protect against proliferative diabetic retinopathy. Exp. Eye Res. 201:108271. doi: 10. 1016/j.exer.2020.108271

Guan, G. Y., Wei, N., Song, T., Zhao, C., Sun, Y., Pan, R. X., et al. (2020). miR-448-3p alleviates diabetic vascular dysfunction by inhibiting endothelialmesenchymal transition through DPP-4 dysregulation. J. Cell. Physiol. 235, 10024-10036. doi: $10.1002 /$ jcp.29817

Guo, R., and Nair, S. (2017). Role of microRNA in diabetic cardiomyopathy: from mechanism to intervention. Biochim. Biophys. Acta Mol. Basis Dis. 1863, 2070-2077. doi: 10.1016/j.bbadis.2017.03.013

Guo, Y., Li, P., Bledsoe, G., Yang, Z.-R., Chao, L., and Chao, J. (2015). Kallistatin inhibits TGF- $\beta$-induced endothelial-mesenchymal transition by differential regulation of microRNA-21 and eNOS expression. Exp. Cell Res. 337, 103-110. doi: 10.1016/j.yexcr.2015.06.021

Gupta, N., Mansoor, S., Sharma, A., Sapkal, A., Sheth, J., Falatoonzadeh, P., et al. (2013). Diabetic retinopathy and VEGF. Open Ophthalmol. J. 7:4.

Hammes, H.-P., Feng, Y., Pfister, F., and Brownlee, M. (2011). Diabetic retinopathy: targeting vasoregression. Diabetes 60, 9-16. doi: 10.2337/db10-0454 
Hansson, G. (2009). Inflammatory mechanisms in atherosclerosis. J. Thromb. Haemostas. 7, 328-331.

Harmanci, D., Erkan, E. P., Kocak, A., and Akdogan, G. G. (2017). Role of the microRNA-29 family in fibrotic skin diseases. Biomed. Rep. 6, 599-604. doi: $10.3892 / \mathrm{br} .2017 .900$

Harries, L. W. (2012). Long non-coding RNAs and human disease. Biochem. Soc. Transact. 40, 902-906.

He, Y., Dan, Y., Gao, X., Huang, L., Lv, H., and Chen, J. (2020). DNMT1-mediated lncRNA MEG3 methylation accelerates endothelial-mesenchymal transition in diabetic retinopathy through the PI3K/AKT/mTOR signaling pathway. Am. J. Physiol. Endocrinol. Metabol. 320, E598-E608.

He, Y., Huang, C., Lin, X., and Li, J. (2013). MicroRNA-29 family, a crucial therapeutic target for fibrosis diseases. Biochimie 95, 1355-1359. doi: 10.1016/j. biochi.2013.03.010

He, Z., Yang, D., Fan, X., Zhang, M., Li, Y., Gu, X., et al. (2020). The roles and mechanisms of lncRNAs in liver fibrosis. Int. J. Mol. Sci. 21:1482. doi: 10.3390/ ijms 21041482

Herrera, J., Henke, C. A., and Bitterman, P. B. (2018). Extracellular matrix as a driver of progressive fibrosis. J. Clin. Investig. 128, 45-53. doi: 10.1172/jci 93557

Hong, L., Du, X., Li, W., Mao, Y., Sun, L., and Li, X. (2018). EndMT: a promising and controversial field. Eur. J. Cell Biol. 97, 493-500. doi: 10.1016/j.ejcb.2018. 07.005

Hortells, L., Johansen, A. K. Z., and Yutzey, K. E. (2019). Cardiac fibroblasts and the extracellular matrix in regenerative and nonregenerative hearts. J. Cardiovasc. Dev. Dis. 6:29. doi: 10.3390/jcdd6030029

Hrenak, J., Paulis, L., and Simko, F. (2015). N-acetyl-seryl-aspartyl-lysylproline (Ac-SDKP): potential target molecule in research of heart, kidney and brain. Curr. Pharmaceut. Design 21, 5135-5143. doi: 10.2174/13816128 21666150909093927

Hu, Q., Li, J., Nitta, K., Kitada, M., Nagai, T., Kanasaki, K., et al. (2018). FGFR1 is essential for N-acetyl-seryl-aspartyl-lysyl-proline regulation of mitochondrial dynamics by upregulating microRNA let-7b-5p. Biochem. Biophys. Res. Commun. 495, 2214-2220. doi: 10.1016/j.bbrc.2017.12.089

Huang, Y., He, Y., and Li, J. (2015). MicroRNA-21: a central regulator of fibrotic diseases via various targets. Curr. Pharmaceut. Design 21, 2236-2242. doi: 10. 2174/1381612820666141226095701

Hulshoff, M. S., del Monte-Nieto, G., Kovacic, J., and Krenning, G. (2019). Noncoding RNA in endothelial-to-mesenchymal transition. Cardiovasc. Res. 115, 1716-1731. doi: 10.1093/cvr/cvz211

Hummel, S. G., Fischer, A. J., Martin, S. M., Schafer, F. Q., and Buettner, G. R. (2006). Nitric oxide as a cellular antioxidant: a little goes a long way. Free Radic. Biol. Med. 40, 501-506. doi: 10.1016/j.freeradbiomed.2005.08.047

Ilhan, F., and Kalkanli, S. T. (2015). Atherosclerosis and the role of immune cells. World J. Clin. Cases WJCC 3:345. doi: 10.12998/wjcc.v3.i4.345

Jahan, F., Landry, N. M., Rattan, S. G., Dixon, I., and Wigle, J. T. (2018). The functional role of zinc finger $\mathrm{E}$ box-binding homeobox 2 (Zeb2) in promoting cardiac fibroblast activation. Int. J. Mol. Sci. 19:3207. doi: 10.3390/ijms19103207

Jia, G., Hill, M. A., and Sowers, J. R. (2018). Diabetic cardiomyopathy: an update of mechanisms contributing to this clinical entity. Circulat. Res. 122, 624-638. doi: $10.1161 /$ circresaha.117.311586

Jiang, L., Hao, C., Li, Z., Zhang, P., Wang, S., Yang, S., et al. (2019). miR-449a induces EndMT, promotes the development of atherosclerosis by targeting the interaction between AdipoR2 and E-cadherin in Lipid Rafts. Biomed. Pharmacother. 109, 2293-2304. doi: 10.1016/j.biopha.2018.11.114

Jiang, Q., Lyu, X.-M., Yuan, Y., and Wang, L. (2017). Plasma miR-21 expression: an indicator for the severity of Type 2 diabetes with diabetic retinopathy. Biosci. Rep. 37:BSR20160589.

Jing, E., Emanuelli, B., Hirschey, M. D., Boucher, J., Lee, K. Y., Lombard, D., et al. (2011). Sirtuin-3 (Sirt3) regulates skeletal muscle metabolism and insulin signaling via altered mitochondrial oxidation and reactive oxygen species production. Proc. Natl. Acad. Sci. 108, 14608-14613. doi: 10.1073/pnas. 1111308108

Joussen, A. M., Poulaki, V., Qin, W., Kirchhof, B., Mitsiades, N., Wiegand, S. J., et al. (2002). Retinal vascular endothelial growth factor induces intercellular adhesion molecule-1 and endothelial nitric oxide synthase expression and initiates early diabetic retinal leukocyte adhesion in vivo. Am. J. Pathol. 160, 501-509. doi: 10.1016/s0002-9440(10)64869-9
Kanasaki, K. (2016). The pathological significance of dipeptidyl peptidase-4 in endothelial cell homeostasis and kidney fibrosis. Diabetol. Int. 7, 212-220. doi: 10.1007/s13340-016-0281-z

Kanasaki, K. (2018). The role of renal dipeptidyl peptidase-4 in kidney disease: renal effects of dipeptidyl peptidase-4 inhibitors with a focus on linagliptin. Clin. Sci. 132, 489-507. doi: 10.1042/cs20180031

Kanasaki, K., Shi, S., Kanasaki, M., He, J., Nagai, T., Nakamura, Y., et al. (2014). Linagliptin-mediated DPP-4 inhibition ameliorates kidney fibrosis in streptozotocin-induced diabetic mice by inhibiting endothelial-tomesenchymal transition in a therapeutic regimen. Diabetes 63, 2120-2131. doi: $10.2337 / \mathrm{db} 13-1029$

Kashihara, N., Haruna, Y., Kondeti, V. K., and Kanwar, Y. S. (2010). Oxidative stress in diabetic nephropathy. Curr. Med. Chem. 17, 4256-4269.

Katakami, N. (2017). Mechanism of development of atherosclerosis and cardiovascular disease in diabetes mellitus. J. Atheroscler. Thromb. 2017:RV17014.

Kaur, H., Chen, S., Xin, X., Chiu, J., Khan, Z. A., and Chakrabarti, S. (2006). Diabetes-induced extracellular matrix protein expression is mediated by transcription coactivator p300. Diabetes 55, 3104-3111. doi: 10.2337/db06-0519

Kehl, T., Backes, C., Kern, F., Fehlmann, T., Ludwig, N., Meese, E., et al. (2017). About miRNAs, miRNA seeds, target genes and target pathways. Oncotarget 8:107167. doi: $10.18632 /$ oncotarget.22363

Kendall, R. T., and Feghali-Bostwick, C. A. (2014). Fibroblasts in fibrosis: novel roles and mediators. Front. Pharmacol. 5:123. doi: 10.3389/fphar.2014.00123

Khaddaj Mallat, R., Mathew John, C., Kendrick, D. J., and Braun, A. P. (2017). The vascular endothelium: A regulator of arterial tone and interface for the immune system. Crit. Rev. Clin. Lab. Sci. 54, 458-470. doi: 10.1080/10408363. 2017.1394267

Kim, J. (2018). MicroRNAs as critical regulators of the endothelial to mesenchymal transition in vascular biology. BMB Rep. 51:65. doi: 10.5483/bmbrep.2018. 51.2.011

Kim, J.-D., Lee, A., Choi, J., Park, Y., Kang, H., Chang, W., et al. (2015). Epigenetic modulation as a therapeutic approach for pulmonary arterial hypertension. Exp. Mol. Med. 47:e175. doi: 10.1038/emm.2015.45

Klaassen, I., Van Noorden, C. J., and Schlingemann, R. O. (2013). Molecular basis of the inner blood-retinal barrier and its breakdown in diabetic macular edema and other pathological conditions. Prog. Retinal Eye Res. 34, 19-48. doi: 10.1016/j.preteyeres.2013.02.001

Klingeborn, M., Dismuke, W. M., Rickman, C. B., and Stamer, W. D. (2017). Roles of exosomes in the normal and diseased eye. Prog. Retinal Eye Res. 59, 158-177. doi: $10.1016 /$ j.preteyeres.2017.04.004

Kolavennu, V., Zeng, L., Peng, H., Wang, Y., and Danesh, F. R. (2008). Targeting of RhoA/ROCK signaling ameliorates progression of diabetic nephropathy independent of glucose control. Diabetes 57, 714-723. doi: 10.2337/db07-1241

Korpal, M., and Kang, Y. (2008). The emerging role of miR-200 family of microRNAs in epithelial-mesenchymal transition and cancer metastasis. RNA Biol. 5, 115-119. doi: 10.4161/rna.5.3.6558

Korpal, M., Lee, E. S., Hu, G., and Kang, Y. (2008). The miR-200 family inhibits epithelial-mesenchymal transition and cancer cell migration by direct targeting of E-cadherin transcriptional repressors ZEB1 and ZEB2. J. Biol. Chem. 283, 14910-14914. doi: 10.1074/jbc.c800074200

Kovacic, J. C., Dimmeler, S., Harvey, R. P., Finkel, T., Aikawa, E., Krenning, G., et al. (2019). Endothelial to mesenchymal transition in cardiovascular disease: JACC state-of-the-art review. J. Am. College Cardiol. 73, 190-209.

Kovacic, J. C., Mercader, N., Torres, M., Boehm, M., and Fuster, V. (2012). Epithelial-to-mesenchymal and endothelial-to-mesenchymal transition: from cardiovascular development to disease. Circulation 125, 1795-1808. doi: 10. 1161/circulationaha.111.040352

Koya, D., and King, G. L. (1998). Protein kinase C activation and the development of diabetic complications. Diabetes 47, 859-866. doi: 10.2337/diabetes.47.6.859

Krenning, G., Barauna, V. G., Krieger, J. E., Harmsen, M. C., and Moonen, J.-R. A. (2016). Endothelial plasticity: shifting phenotypes through force feedback. Stem Cells Int. 2016:9762959.

Kriegel, A. J., Liu, Y., Fang, Y., Ding, X., and Liang, M. (2012). The miR-29 family: genomics, cell biology, and relevance to renal and cardiovascular injury. Physiol. Genomics 44, 237-244. doi: 10.1152/physiolgenomics.00141.2011

Kumarswamy, R., Volkmann, I., Jazbutyte, V., Dangwal, S., Park, D.-H., and Thum, T. (2012). Transforming growth factor- $\beta$-induced endothelial-to-mesenchymal 
transition is partly mediated by microRNA-21. Arterioscler. Thromb. Vascul. Biol. 32, 361-369. doi: 10.1161/atvbaha.111.234286

Kusuhara, S., Fukushima, Y., Ogura, S., Inoue, N., and Uemura, A. (2018). Pathophysiology of diabetic retinopathy: the old and the new. Diab. Metabol. J. 42:364. doi: $10.4093 / \mathrm{dmj} .2018 .0182$

La Sala, L., Mrakic-Sposta, S., Micheloni, S., Prattichizzo, F., and Ceriello, A. (2018). Glucose-sensing microRNA-21 disrupts ROS homeostasis and impairs antioxidant responses in cellular glucose variability. Cardiovasc. Diabetol. 17, 1-14. doi: 10.2174/978160805189211101010001

La Sala, L., Mrakic-Sposta, S., Tagliabue, E., Prattichizzo, F., Micheloni, S., Sangalli, E., et al. (2019a). Circulating microRNA-21 is an early predictor of ROSmediated damage in subjects with high risk of developing diabetes and in drugnaïve T2D. Cardiovasc. Diabetol. 18, 1-12. doi: 10.2165/11533370-00000000000000

La Sala, L., Prattichizzo, F., and Ceriello, A. (2019b). The link between diabetes and atherosclerosis. Eur. J. Prevent. Cardiol. 26, 15-24.

Lechner, J., O'Leary, O. E., and Stitt, A. W. (2017). The pathology associated with diabetic retinopathy. Vis. Res. 139, 7-14. doi: 10.1016/j.visres.2017. 04.003

Lee, R., Wong, T. Y., and Sabanayagam, C. (2015). Epidemiology of diabetic retinopathy, diabetic macular edema and related vision loss. Eye Vis. 2, 1-25.

Leung, A., and Natarajan, R. (2018). Long noncoding RNAs in diabetes and diabetic complications. Antioxid. Redox Signal. 29, 1064-1073. doi: 10.1089/ars.2017. 7315

Lewis, G., and Maxwell, A. P. (2014). Risk factor control is key in diabetic nephropathy. Practitioner 258, 13-17.

Li, J., Cao, L.-T., Liu, H.-H., Yin, X.-D., and Wang, J. (2020a). Long non coding RNA H19: An emerging therapeutic target in fibrosing diseases. Autoimmunity 53, 1-7. doi: 10.1080/08916934.2019.1681983

Li, J., Liu, H., Srivastava, S. P., Hu, Q., Gao, R., Li, S., et al. (2020b). Endothelial FGFR1 (fibroblast growth factor receptor 1) deficiency contributes differential fibrogenic effects in kidney and heart of diabetic mice. Hypertension 76, 19351944. doi: 10.1161/hypertensionaha.120.15587

Li, Q., Lin, Y., Wang, S., Zhang, L., and Guo, L. (2017). GLP-1 inhibits high-glucose-induced oxidative injury of vascular endothelial cells. Sci. Rep. $7,1-9$.

Li, Q., Yao, Y., Shi, S., Zhou, M., Zhou, Y., Wang, M., et al. (2020). Inhibition of miR-21 alleviated cardiac perivascular fibrosis via repressing EndMT in T1DM. J. Cell. Mol. Med. 24, 910-920. doi: 10.1111/jcmm.14800

Liang, C., Gao, L., Liu, Y., Liu, Y., Yao, R., Li, Y., et al. (2019). MiR-451 antagonist protects against cardiac fibrosis in streptozotocin-induced diabetic mouse heart. Life Sci. 224, 12-22. doi: 10.1016/j.lfs.2019.02.059

Lim, A. K. (2014). Diabetic nephropathy-complications and treatment. Int. J. Nephrol. Renovasc. Dis. 7:361. doi: 10.2147/ijnrd.s40172

Lin, J., Jiang, Z., Liu, C., Zhou, D., Song, J., Liao, Y., et al. (2020). Emerging Roles of Long Non-Coding RNAs in Renal Fibrosis. Life 10:131. doi: 10.3390/ life10080131

Lin, Q., Zhao, J., Zheng, C., and Chun, J. (2018). Roles of notch signaling pathway and endothelial-mesenchymal transition in vascular endothelial dysfunction and atherosclerosis. Eur. Rev. Med. Pharmacol. Sci. 22, 6485-6491.

Lin, X., Xu, Y., Pan, X., Xu, J., Ding, Y., Sun, X., et al. (2020). Global, regional, and national burden and trend of diabetes in 195 countries and territories: an analysis from 1990 to 2025. Sci. Rep. 10, 1-11.

Liu, F., Zhang, S., Xu, R., Gao, S., and Yin, J. (2018). Melatonin attenuates endothelial-to-mesenchymal transition of glomerular endothelial cells via regulating miR-497/ROCK in diabetic nephropathy. Kidney Blood Pressure Res. 43, 1425-1436. doi: 10.1159/000493380

Liu, J., Jiang, F., Jiang, Y., Wang, Y., Li, Z., Shi, X., et al. (2020). Roles of Exosomes in Ocular Diseases. Int. J. Nanomed. 15:10519. doi: 10.2147/ijn.s277190

Liu, L., and Liu, X. (2019). Roles of drug transporters in blood-retinal barrier. Drug Transporters in Drug Disposition. Effects Toxicity 2019, 467-504. doi: 10.1007/978-981-13-7647-4_10

Liu, R. H., Ning, B., Ma, X. E., Gong, W. M., and Jia, T. H. (2016). Regulatory roles of microRNA-21 in fibrosis through interaction with diverse pathways. Mol. Med. Rep. 13, 2359-2366. doi: 10.3892/mmr.2016.4834

Lnsis, A. (2000). Atherosclenrosis. Nature 407, 233-241.

Loboda, A., Sobczak, M., Jozkowicz, A., and Dulak, J. (2016). TGF- $\beta 1 /$ Smads and miR-21 in renal fibrosis and inflammation. Mediat. Inflamm. 2016:8319283.
Locatelli, M., Zoja, C., Zanchi, C., Corna, D., Villa, S., Bolognini, S., et al. (2020). Manipulating Sirtuin 3 pathway ameliorates renal damage in experimental diabetes. Sci. Rep. 10, 1-12. doi: 10.1155/2015/780903

Luc, K., Schramm-Luc, A., Guzik, T., and Mikolajczyk, T. (2019). Oxidative stress and inflammatory markers in prediabetes and diabetes. J. Physiol. Pharmacol. $70,809-824$.

Ma, F., Xu, S., Liu, X., Zhang, Q., Xu, X., Liu, M., et al. (2011). The microRNA miR29 controls innate and adaptive immune responses to intracellular bacterial infection by targeting interferon- $\gamma$. Nat. Immunol. 12, 861-869. doi: 10.1038/ ni.2073

Ma, Z.-G., Yuan, Y.-P., Wu, H.-M., Zhang, X., and Tang, Q.-Z. (2018). Cardiac fibrosis: new insights into the pathogenesis. Int. J. Biol. Sci. 14:1645. doi: 10. 7150/ijbs. 28103

Maghbooli, Z., Hossein-nezhad, A., Larijani, B., Amini, M., and Keshtkar, A. (2015). Global DNA methylation as a possible biomarker for diabetic retinopathy. Diab. Metabol. Res. Rev. 31, 183-189. doi: 10.1002/dmrr.2584

Man, S., Duffhues, G. S., Ten Dijke, P., and Baker, D. (2019). The therapeutic potential of targeting the endothelial-to-mesenchymal transition. Angiogenesis 22, 3-13. doi: 10.1007/s10456-018-9639-0

Mangiafico, S. P., Lim, S. H., Neoh, S., Massinet, H., Joannides, C. N., Proietto, J., et al. (2011). A primary defect in glucose production alone cannot induce glucose intolerance without defects in insulin secretion. J. Endocrinol. 210:335. doi: 10.1530/joe-11-0126

Matoba, K., Takeda, Y., Nagai, Y., Kanazawa, Y., Kawanami, D., Yokota, T., et al. (2020). ROCK Inhibition May Stop Diabetic Kidney Disease. JMA J. 3, 154-163. doi: 10.31662/jmaj.2020-0014

McArthur, K., Feng, B., Wu, Y., Chen, S., and Chakrabarti, S. (2011). MicroRNA$200 \mathrm{~b}$ regulates vascular endothelial growth factor-mediated alterations in diabetic retinopathy. Diabetes 60, 1314-1323. doi: 10.2337/db10-1557

Meadows, K. N., Iyer, S., Stevens, M. V., Wang, D., Shechter, S., Perruzzi, C., et al. (2009). Akt promotes endocardial-mesenchyme transition. J. Angiogene. Res. $1,1-9$.

Medici, D., Potenta, S., and Kalluri, R. (2011). Transforming growth factor$\beta 2$ promotes Snail-mediated endothelial-mesenchymal transition through convergence of Smad-dependent and Smad-independent signalling. Biochem. J. 437, 515-520. doi: 10.1042/bj20101500

Meza, C. A., La Favor, J. D., Kim, D.-H., and Hickner, R. C. (2019). Endothelial dysfunction: is there a hyperglycemia-induced imbalance of NOX and NOS? Int. J. Mol. Sci. 20:3775. doi: 10.3390/ijms20153775

Micallef, L., Vedrenne, N., Billet, F., Coulomb, B., Darby, I. A., and Desmoulière, A. (2012). The myofibroblast, multiple origins for major roles in normal and pathological tissue repair. Fibrogene. Tissue Repair 5(Suppl. 1):S5.

Michlewski, G., and Cáceres, J. F. (2019). Post-transcriptional control of miRNA biogenesis. RNA 25, 1-16. doi: 10.1261/rna.068692.118

Mima, A. (2013). Inflammation and oxidative stress in diabetic nephropathy: new insights on its inhibition as new therapeutic targets. J. Diab. Res. 2013:248563.

Mogensen, C., Christensen, C., and Vittinghus, E. (1983). The stages in diabetic renal disease: with emphasis on the stage of incipient diabetic nephropathy. Diabetes 32(Suppl. 2), 64-78. doi: 10.2337/diab.32.2.s64

Montorfano, I., Becerra, A., Cerro, R., Echeverría, C., Sáez, E., Morales, M. G., et al. (2014). Oxidative stress mediates the conversion of endothelial cells into myofibroblasts via a TGF- $\beta 1$ and TGF- $\beta$ 2-dependent pathway. Lab. Investig. 94, 1068-1082. doi: 10.1038/labinvest.2014.100

Moreno-Viedma, V., Amor, M., Sarabi, A., Bilban, M., Staffler, G., Zeyda, M., et al. (2016). Common dysregulated pathways in obese adipose tissue and atherosclerosis. Cardiovasc. Diabetol. 15, 1-12.

Nagai, T., Kanasaki, M., Srivastava, S. P., Nakamura, Y., Ishigaki, Y., Kitada, M., et al. (2014). N-acetyl-seryl-aspartyl-lysyl-proline inhibits diabetes-associated kidney fibrosis and endothelial-mesenchymal transition. BioMed Res. Int. 2014:696475.

Nandi, S. S., and Mishra, P. K. (2018). Targeting miRNA for therapy of juvenile and adult diabetic cardiomyopathy. Exosomes, Stem Cells and MicroRNA. Berlin: Springer, 47-59.

Nemir, M., Metrich, M., Plaisance, I., Lepore, M., Cruchet, S., Berthonneche, C., et al. (2014). The Notch pathway controls fibrotic and regenerative repair in the adult heart. Eur. Heart J. 35, 2174-2185. doi: 10.1093/eurheartj/ ehs269 
Nithianandarajah-Jones, G. N., Wilm, B., Goldring, C. E., Müller, J., and Cross, M. J. (2014). The role of ERK5 in endothelial cell function. Biochem. Soc. Transact. 42, 1584-1589. doi: 10.1042/bst20140276

Nitta, K., Shi, S., Nagai, T., Kanasaki, M., Kitada, M., Srivastava, S. P., et al. (2016). Oral administration of $\mathrm{N}$-acetyl-seryl-aspartyl-lysyl-proline ameliorates kidney disease in both type 1 and type 2 diabetic mice via a therapeutic regimen. BioMed Res. Int. 2016:9172157.

Noseda, M., McLean, G., Niessen, K., Chang, L., Pollet, I., Montpetit, R., et al. (2004). Notch activation results in phenotypic and functional changes consistent with endothelial-to-mesenchymal transformation. Circulat. Res. 94, 910-917. doi: 10.1161/01.res.0000124300.76171.c9

Oguntibeju, O. O. (2019). Type 2 diabetes mellitus, oxidative stress and inflammation: examining the links. Int. J. Physiol. Pathophysiol. Pharmacol. 11:45.

Pardali, E., Sanchez-Duffhues, G., Gomez-Puerto, M. C., and Ten Dijke, P. (2017). TGF- $\beta$-induced endothelial-mesenchymal transition in fibrotic diseases. Int. J. Mol. Sci. 18:2157. doi: 10.3390/ijms18102157

Patel, S., Rauf, A., Khan, H., and Abu-Izneid, T. (2017). Renin-angiotensinaldosterone (RAAS): The ubiquitous system for homeostasis and pathologies. Biomed. Pharmacother. 94, 317-325. doi: 10.1016/j.biopha.2017.07.091

Pathak, J. V., and Dass, E. E. (2015). A retrospective study of the effects of angiotensin receptor blockers and angiotensin converting enzyme inhibitors in diabetic nephropathy. Ind. J. Pharmacol. 47:148. doi: 10.4103/0253-7613. 153420

Peng, C., Ma, J., Gao, X., Tian, P., Li, W., and Zhang, L. (2013). High glucose induced oxidative stress and apoptosis in cardiac microvascular endothelial cells are regulated by FoxO3a. PLoS One 8:e79739. doi: 10.1371/journal.pone. 0079739

Phan, T. H. G., Paliogiannis, P., Nasrallah, G. K., Giordo, R., Eid, A. H., Fois, A. G., et al. (2020). Emerging cellular and molecular determinants of idiopathic pulmonary fibrosis. Cell. Mol. Life Sci. 2020, 1-27.

Piera-Velazquez, S., and Jimenez, S. A. (2019). Endothelial to mesenchymal transition: role in physiology and in the pathogenesis of human diseases. Physiol. Rev. 99, 1281-1324. doi: 10.1152/physrev.00021.2018

Poznyak, A., Grechko, A. V., Poggio, P., Myasoedova, V. A., Alfieri, V., and Orekhov, A. N. (2020). The diabetes mellitus-atherosclerosis connection: The role of lipid and glucose metabolism and chronic inflammation. Int. J. Mol. Sci. 21:1835. doi: $10.3390 /$ ijms21051835

Qadir, M. M. F., Klein, D., Álvarez-Cubela, S., Domínguez-Bendala, J., and Pastori, R. L. (2019). The role of microRNAs in diabetes-related oxidative stress. Int. J. Mol. Sci. 20:5423. doi: 10.3390/ijms20215423

Qiu, X., Brown, K., Hirschey, M. D., Verdin, E., and Chen, D. (2010). Calorie restriction reduces oxidative stress by SIRT3-mediated SOD2 activation. Cell Metabol. 12, 662-667. doi: 10.1016/j.cmet.2010.11.015

Qu, S., Yang, X., Li, X., Wang, J., Gao, Y., Shang, R., et al. (2015). Circular RNA: a new star of noncoding RNAs. Cancer Lett. 365, 141-148. doi: 10.1016/j.canlet. 2015.06.003

Qu, S., Zhong, Y., Shang, R., Zhang, X., Song, W., Kjems, J., et al. (2017). The emerging landscape of circular RNA in life processes. RNA Biol. 14, 992-999. doi: 10.1080/15476286.2016.1220473

Ray, D., Mishra, M., Ralph, S., Read, I., Davies, R., and Brenchley, P. (2004). Association of the VEGF gene with proliferative diabetic retinopathy but not proteinuria in diabetes. Diabetes 53, 861-864. doi: 10.2337/diabetes.53.3.861

Reddy, K. J., Singh, M., Bangit, J. R., and Batsell, R. R. (2010). The role of insulin resistance in the pathogenesis of atherosclerotic cardiovascular disease: an updated review. J. Cardiovasc. Med. 11, 633-647. doi: 10.2459/jcm. 0b013e328333645a

Regazzi, R. (2018). MicroRNAs as therapeutic targets for the treatment of diabetes mellitus and its complications. Expert Opin. Therapeut. Targets 22, 153-160. doi: 10.1080/14728222.2018.1420168

Rhee, S. Y., and Kim, Y. S. (2018). The role of advanced glycation end products in diabetic vascular complications. Diab. Metabol. J. 42:188. doi: 10.4093/dmj. 2017.0105

Rheinberger, M., and Böger, C. (2014). Diabetic nephropathy: new insights into diagnosis, prevention and treatment. Deutsche Medizinische Wochenschrift 139, 704-706.

Romero-Aroca, P., Baget-Bernaldiz, M., Pareja-Rios, A., Lopez-Galvez, M., Navarro-Gil, R., and Verges, R. (2016). Diabetic macular edema pathophysiology: vasogenic versus inflammatory. J. Diab. Res. 2016: 2156273.

Roy, S., Bae, E., Amin, S., and Kim, D. (2015). Extracellular matrix, gap junctions, and retinal vascular homeostasis in diabetic retinopathy. Exp. Eye Res. 133, 58-68. doi: 10.1016/j.exer.2014.08.011

Ruiz-Ortega, M., Rodrigues-Diez, R. R., Lavoz, C., and Rayego-Mateos, S. (2020). Special issue "diabetic nephropathy: Diagnosis, prevention and treatment". Basel: Multidisciplinary Digital Publishing Institute.

Ruknarong, L., Boonthongkaew, C., Chuangchot, N., Jumnainsong, A., Leelayuwat, N., Jusakul, A., et al. (2021). Vitamin C supplementation reduces expression of circulating miR-45la in subjects with poorly controlled type 2 diabetes mellitus and high oxidative stress. PeerJ 9:e10776. doi: 10.7717/peerj.10776

Russell, J. W., Golovoy, D., Vincent, A. M., Mahendru, P., Olzmann, J. A., Mentzer, A., et al. (2002). High glucose-induced oxidative stress and mitochondrial dysfunction in neurons. FASEB J. 16, 1738-1748. doi: 10.1096/fj.01$1027 \mathrm{com}$

Sabanayagam, C., Yip, W., Ting, D. S., Tan, G., and Wong, T. Y. (2016). Ten emerging trends in the epidemiology of diabetic retinopathy. Ophthal. Epidemiol. 23, 209-222. doi: 10.1080/09286586.2016.1193618

Sánchez-Duffhues, G., García de Vinuesa, A., and Ten Dijke, P. (2018). Endothelial-to-mesenchymal transition in cardiovascular diseases: developmental signaling pathways gone awry. Dev. Dynam. 247, 492-508. doi: $10.1002 /$ dvdy.24589

Sandoo, A., van Zanten, J. J. V., Metsios, G. S., Carroll, D., and Kitas, G. D. (2010). The endothelium and its role in regulating vascular tone. Open Cardiovasc. Med. J. 4:302. doi: 10.2174/1874192401004010302

Schiffrin, E. L. (2008). Oxidative stress, nitric oxide synthase, and superoxide dismutase: a matter of imbalance underlies endothelial dysfunction in the human coronary circulation. Hypertension 51, 31-32. doi: 10.1161/ hypertensionaha.107.103226

Semenkovich, C. F. (2006). Insulin resistance and atherosclerosis. J. Clin. Investig. $116,1813-1822$.

Sharma, V., Dogra, N., Saikia, U. N., and Khullar, M. (2017). Transcriptional regulation of endothelial-to-mesenchymal transition in cardiac fibrosis: role of myocardin-related transcription factor A and activating transcription factor 3. Canad. J. Physiol. Pharmacol. 95, 1263-1270. doi: 10.1139/cjpp-20160634

Shaw, J. E., Sicree, R. A., and Zimmet, P. Z. (2010). Global estimates of the prevalence of diabetes for 2010 and 2030. Diab. Res. Clin. Pract. 87, 4-14. doi: 10.1016/j.diabres.2009.10.007

Shi, S., Song, L., Yu, H., Feng, S., He, J., Liu, Y., et al. (2020). Knockdown of LncRNA-H19 Ameliorates Kidney Fibrosis in Diabetic Mice by Suppressing miR-29a-Mediated EndMT. Front. Pharmacol. 11:1936. doi: 10.3389/fphar. 2020.586895

Shi, S., Srivastava, S. P., Kanasaki, M., He, J., Kitada, M., Nagai, T., et al. (2015). Interactions of DPP- 4 and integrin $\beta 1$ influences endothelial-to-mesenchymal transition. Kidney Int. 88, 479-489. doi: 10.1038/ki.2015.103

Shi, X., Sun, M., Liu, H., Yao, Y., and Song, Y. (2013). Long non-coding RNAs: a new frontier in the study of human diseases. Cancer Lett. 339, 159-166. doi: 10.1016/j.canlet.2013.06.013

Siasos, G., Tsigkou, V., Kosmopoulos, M., Theodosiadis, D., Simantiris, S., Tagkou, N. M., et al. (2018). Mitochondria and cardiovascular diseases-from pathophysiology to treatment. Ann. Translat. Med. 6:256.

Souilhol, C., Harmsen, M. C., Evans, P. C., and Krenning, G. (2018). Endothelialmesenchymal transition in atherosclerosis. Cardiovasc. Res. 114, 565-577. doi: $10.1093 / \mathrm{cvr} / \mathrm{cvx} 253$

Srivastava, S. P., Goodwin, J. E., Kanasaki, K., and Koya, D. (2020a). Inhibition of angiotensin-converting enzyme ameliorates renal fibrosis by mitigating dpp-4 level and restoring antifibrotic micrornas. Genes 11:211. doi: 10.3390/ genes11020211

Srivastava, S. P., Goodwin, J. E., Kanasaki, K., and Koya, D. (2020b). Metabolic reprogramming by $\mathrm{N}$-acetyl-seryl-aspartyl-lysyl-proline protects against diabetic kidney disease. Br. J. Pharmacol. 177, 3691-3711. doi: 10.1111/ bph. 15087

Srivastava, S. P., Hedayat, A. F., Kanasaki, K., and Goodwin, J. E. (2019). Microrna crosstalk influences epithelial-to-mesenchymal, endothelial-to-mesenchymal, and macrophage-to-mesenchymal transitions in the kidney. Front. Pharmacol. 10:904. doi: 10.3389/fphar.2019.00904 
Srivastava, S. P., Kanasaki, K., and Goodwin, J. E. (2020c). Loss of Mitochondrial Control Impacts Renal Health. Front. Pharmacol. 11:2133. doi: 10.3389/fphar. 2020.543973

Srivastava, S. P., Koya, D., and Kanasaki, K. (2013). MicroRNAs in kidney fibrosis and diabetic nephropathy: roles on EMT and EndMT. BioMed Res. Int. 2013:125469.

Srivastava, S. P., Li, J., Kitada, M., Fujita, H., Yamada, Y., Goodwin, J. E., et al. (2018). SIRT3 deficiency leads to induction of abnormal glycolysis in diabetic kidney with fibrosis. Cell Death Dis. 9, 1-14.

Srivastava, S. P., Shi, S., Kanasaki, M., Nagai, T., Kitada, M., He, J., et al. (2016). Effect of antifibrotic microRNAs crosstalk on the action of $\mathrm{N}$-acetyl-serylaspartyl-lysyl-proline in diabetes-related kidney fibrosis. Sci. Rep. 6, 1-12. doi: $10.1155 / 2014 / 696475$

Statello, L., Guo, C.-J., Chen, L.-L., and Huarte, M. (2020). Gene regulation by long non-coding RNAs and its biological functions. Nat. Rev. Mol. Cell Biol. 2020, 1-23. doi: 10.1007/978-3-030-17086-8_1

Su, Q., Sun, Y., Ye, Z., Yang, H., and Li, L. (2018). Oxidized low density lipoprotein induces endothelial-to-mesenchymal transition by stabilizing snail in human aortic endothelial cells. Biomed. Pharmacother. 106, 1720-1726. doi: 10.1016/j. biopha.2018.07.122

Sun, Y. B. Y., Qu, X., Caruana, G., and Li, J. (2016). The origin of renal fibroblasts/myofibroblasts and the signals that trigger fibrosis. Differentiation 92, 102-107. doi: 10.1016/j.diff.2016.05.008

Taft, R. J., Pang, K. C., Mercer, T. R., Dinger, M., and Mattick, J. S. (2010). Noncoding RNAs: regulators of disease. J. Pathol. J. Pathol. Soc. Great Br. Irel. 220, 126-139. doi: 10.1002/path.2638

Tan, K. C., Chow, W.-S., Ai, V. H., Metz, C., Bucala, R., and Lam, K. S. (2002). Advanced glycation end products and endothelial dysfunction in type 2 diabetes. Diab. Care 25, 1055-1059. doi: 10.2337/diacare.25.6. 1055

Tan, Y., Zhang, Z., Zheng, C., Wintergerst, K. A., Keller, B. B., and Cai, L. (2020). Mechanisms of diabetic cardiomyopathy and potential therapeutic strategies: preclinical and clinical evidence. Nat. Rev. Cardiol. 17, 585-607. doi: 10.1038/ s41569-020-0339-2

Tao, H., Yang, J.-J., Shi, K.-H., and Li, J. (2016). Wnt signaling pathway in cardiac fibrosis: new insights and directions. Metabolism 65, 30-40. doi: 10.1016/j. metabol.2015.10.013

Tesfamariam, B. (1994). Free radicals in diabetic endothelial cell dysfunction. Free Radic. Biol. Med. 16, 383-391. doi: 10.1016/0891-5849(94)90040-x

Thomas, A. A., Biswas, S., Feng, B., Chen, S., Gonder, J., and Chakrabarti, S. (2019). IncRNA H19 prevents endothelial-mesenchymal transition in diabetic retinopathy. Diabetologia 62, 517-530. doi: 10.1007/s00125-0184797-6

Thuan, D. T. B., Zayed, H., Eid, A. H., Abou-Saleh, H., Nasrallah, G. K., Mangoni, A. A., et al. (2018). A potential link between oxidative stress and endothelialto-mesenchymal transition in systemic sclerosis. Front. Immunol. 9:1985. doi: 10.3389/fimmu.2018.01985

Tian, D.-Y., Jin, X.-R., Zeng, X., and Wang, Y. (2017). Notch signaling in endothelial cells: is it the therapeutic target for vascular neointimal hyperplasia? Int. J. Mol. Sci. 18:1615. doi: 10.3390/ijms18081615

Trost, A., Bruckner, D., Rivera, F. J., and Reitsamer, H. A. (2019). Pericytes in the Retina. Pericyte Biol. Differ. Organs 1122, 1-26.

Van Geest, R. J., Klaassen, I., Vogels, I. M., Van Noorden, C. J., and Schlingemann, R. O. (2010). Differential TGF- $\beta$ signaling in retinal vascular cells: a role in diabetic retinopathy? Investigat. Ophthalmol. Vis. Sci. 51, 1857-1865. doi: 10. 1167/iovs.09-4181

Vanchin, B., Offringa, E., Friedrich, J., Brinker, M. G., Kiers, B., Pereira, A. C., et al. (2019). MicroRNA-374b induces endothelial-to-mesenchymal transition and early lesion formation through the inhibition of MAPK7 signaling. J. Pathol. 247, 456-470. doi: 10.1002/path.5204

Vanessa Fiorentino, T., Prioletta, A., Zuo, P., and Folli, F. (2013). Hyperglycemia-induced oxidative stress and its role in diabetes mellitus related cardiovascular diseases. Curr. Pharmaceut. Design 19, 5695-5703. doi: 10.2174/1381612811319320005

Verjans, R., Peters, T., Beaumont, F. J., van Leeuwen, R., van Herwaarden, T., Verhesen, W., et al. (2018). MicroRNA-221/222 family counteracts myocardial fibrosis in pressure overload-induced heart failure. Hypertension 71, 280-288. doi: 10.1161/hypertensionaha.117.10094
Vidigal, J. A., and Ventura, A. (2015). The biological functions of miRNAs: lessons from in vivo studies. Trends Cell Biol. 25, 137-147. doi: 10.1016/j.tcb.2014. 11.004

Volpe, C. M. O., Villar-Delfino, P. H., Dos Anjos, P. M. F., and NogueiraMachado, J. A. (2018). Cellular death, reactive oxygen species (ROS) and diabetic complications. Cell Death Dis. 9, 1-9.

Wang, C., Wang, L., Ding, Y., Lu, X., Zhang, G., Yang, J., et al. (2017). LncRNA structural characteristics in epigenetic regulation. Int. J. Mol. Sci. 18:2659. doi: 10.3390/ijms18122659

Wang, H. H., Garruti, G., Liu, M., Portincasa, P., and Wang, D. Q.-H. (2017). Cholesterol and lipoprotein metabolism and atherosclerosis: recent advances in reverse cholesterol transport. Ann. Hepatol. 16, S27-S42.

Wang, H., and Cai, J. (2017). The role of microRNAs in heart failure. Biochim. Biophys. Acta Mol. Basis Dis. 1863, 2019-2030.

Wang, J., Liew, O. W., Richards, A. M., and Chen, Y.-T. (2016). Overview of microRNAs in cardiac hypertrophy, fibrosis, and apoptosis. Int. J. Mol. Sci. 17:749. doi: 10.3390/ijms17050749

Wang, W., and Lo, A. C. (2018). Diabetic retinopathy: pathophysiology and treatments. Int. J. Mol. Sci. 19:1816. doi: 10.3390/ijms19061816

Wang, Z., Han, Z., Tao, J., Wang, J., Liu, X., Zhou, W., et al. (2017). Role of endothelial-to-mesenchymal transition induced by TGF- $\beta 1$ in transplant kidney interstitial fibrosis. J. Cell. Mol. Med. 21, 2359-2369. doi: 10.1111/jcmm. 13157

Wang, Z., Han, Z., Tao, J., Wang, J., Liu, X., Zhou, W., et al. (2016). Transforming growth factor- $\beta 1$ induces endothelial-to-mesenchymal transition via Akt signaling pathway in renal transplant recipients with chronic allograft dysfunction. Medical Sci. Monit. 21, 775-783. doi: 10.12659/aot.899931

Wang, Z., Wang, Z., Gao, L., Xiao, L., Yao, R., Du, B., et al. (2020). miR-222 inhibits cardiac fibrosis in diabetic mice heart via regulating $W n t / \beta$-catenin-mediated endothelium to mesenchymal transition. J. Cell. Physiol. 235, 2149-2160. doi: $10.1002 /$ jcp.29119

Wellen, K. E., and Hotamisligil, G. S. (2005). Inflammation, stress, and diabetes. J. Clin. Investig. 115, 1111-1119.

Widyantoro, B., Emoto, N., Nakayama, K., Anggrahini, D. W., Adiarto, S., Iwasa, N., et al. (2010). Endothelial cell-derived endothelin-1 promotes cardiac fibrosis in diabetic heart through endothelial-to-mesenchymal transition. Circulation 121, 2407-2418. doi: 10.1161/circulationaha.110.938217

Williams, V. R., and Scholey, J. W. (2018). Angiotensin-converting enzyme 2 and renal disease. Curr. Opin. Nephrol. Hypertens. 27, 35-41.

Wu, H., Liu, T., and Hou, H. (2020). Knockdown of LINC00657 inhibits ox-LDLinduced endothelial cell injury by regulating miR-30c-5p/Wnt $7 \mathrm{~b} / \beta$-catenin. Mol. Cell. Biochem. 472, 145-155. doi: 10.1007/s11010-020-03793-9

Wu, X., Du, X., Yang, Y., Liu, X., Liu, X., Zhang, N., et al. (2021). Inhibition of miR122 reduced atherosclerotic lesion formation by regulating NPAS3-mediated endothelial to mesenchymal transition. Life Sci. 265:118816. doi: 10.1016/j.lfs. 2020.118816

Wynn, T. A. (2008). Cellular and molecular mechanisms of fibrosis. J. Pathol. J. Pathol. Soc. Great Br. Irel. 214, 199-210.

Yamagishi, S.-I., and Matsui, T. (2018). Role of hyperglycemia-induced advanced glycation end product (AGE) accumulation in atherosclerosis. Ann. Vasc. Dis. 11, 253-258. doi: 10.3400/avd.ra.18-00070

Yan, D., Luo, X., Li, Y., Liu, W., Deng, J., Zheng, N., et al. (2014). Effects of advanced glycation end products on calcium handling in cardiomyocytes. Cardiology 129, 75-83. doi: 10.1159/000364779

Yang, L., Kwon, J., Popov, Y., Gajdos, G. B., Ordog, T., Brekken, R. A., et al. (2014). Vascular endothelial growth factor promotes fibrosis resolution and repair in mice. Gastroenterology 146, 1339-1350.

Yang, S., Banerjee, S., de Freitas, A., Sanders, Y. Y., Ding, Q., Matalon, S., et al. (2012). Participation of miR-200 in pulmonary fibrosis. Am. J. Pathol. 180, 484-493. doi: 10.1016/j.ajpath.2011.10.005

Yildirim, S. S., Akman, D., Catalucci, D., and Turan, B. (2013). Relationship between downregulation of miRNAs and increase of oxidative stress in the development of diabetic cardiac dysfunction: junctin as a target protein of miR-1. Cell Biochem. Biophys. 67, 1397-1408. doi: 10.1007/s12013-0139672-y

Ying, W. (2008). NAD+/NADH and NADP+/NADPH in cellular functions and cell death: regulation and biological consequences. Antioxid. Redox Signal. 10, 179-206. doi: 10.1089/ars.2007.1672 
Yousefi, F., Shabaninejad, Z., Vakili, S., Derakhshan, M., Movahedpour, A., Dabiri, H., et al. (2020). TGF- $\beta$ and WNT signaling pathways in cardiac fibrosis: non-coding RNAs come into focus. Cell Commun. Signal. 18, 1-16.

Yu, C.-H., Gong, M., Liu, W.-J., Cui, N.-X., Wang, Y., Du, X., et al. (2017). High glucose induced endothelial to mesenchymal transition in human umbilical vein endothelial cell. Exp. Mol. Pathol. 102, 377-383. doi: 10.1016/j.yexmp.2017. 03.007

Yu, T., Jhun, B. S., and Yoon, Y. (2011). High-glucose stimulation increases reactive oxygen species production through the calcium and mitogen-activated protein kinase-mediated activation of mitochondrial fission. Antioxid. Redox Signal. 14, 425-437. doi: 10.1089/ars.2010.3284

Yuan, J., Chen, H., Ge, D., Xu, Y., Xu, H., Yang, Y., et al. (2017). Mir-21 promotes cardiac fibrosis after myocardial infarction via targeting Smad7. Cell. Physiol. Biochem. 42, 2207-2219. doi: 10.1159/000479995

Yuan, T., Yang, T., Chen, H., Fu, D., Hu, Y., Wang, J., et al. (2019). New insights into oxidative stress and inflammation during diabetes mellitus-accelerated atherosclerosis. Redox Biol. 20, 247-260. doi: 10.1016/j.redox.2018.09.025

Yue, B. (2014). Biology of the extracellular matrix: an overview. J. Glaucoma 23(8 Suppl. 1), S20-S23.

Zeisberg, E. M., Potenta, S. E., Sugimoto, H., Zeisberg, M., and Kalluri, R. (2008). Fibroblasts in kidney fibrosis emerge via endothelial-to-mesenchymal transition. J. Am. Soc. Nephrol. 19, 2282-2287. doi: 10.1681/asn.2008050513

Zent, J., and Guo, L.-W. (2018). Signaling mechanisms of myofibroblastic activation: outside-in and inside-out. Cell. Physiol. Biochem. 49, 848-868. doi: $10.1159 / 000493217$

Zhang, H., Feng, Z., Huang, R., Xia, Z., Xiang, G., and Zhang, J. (2014). MicroRNA449 suppresses proliferation of hepatoma cell lines through blockade lipid metabolic pathway related to SIRT1. Int. J. Oncol. 45, 2143-2152. doi: 10.3892/ ijo. 2014.2596

Zhang, H., Hu, J., and Liu, L. (2017). MiR-200a modulates TGF- $\beta 1$-induced endothelial-to-mesenchymal shift via suppression of GRB2 in HAECs. Biomed. Pharmacother. 95, 215-222. doi: 10.1016/j.biopha.2017.07.104
Zhang, J., Zeng, Y., Chen, J., Cai, D., Chen, C., Zhang, S., et al. (2019). miR-29a/b cluster suppresses high glucose-induced endothelial-mesenchymal transition in human retinal microvascular endothelial cells by targeting Notch2. Exp. Therapeut. Med. 17, 3108-3116.

Zhang, Y., Huang, X.-R., Wei, L.-H., Chung, A. C., Yu, C.-M., and Lan, H.Y. (2014). miR-29b as a therapeutic agent for angiotensin II-induced cardiac fibrosis by targeting TGF- $\beta /$ Smad3 signaling. Mol. Therap. 22, 974-985. doi: 10.1038/mt.2014.25

Zhao, Y., Zhang, J., Li, H., Li, Y., Ren, J., Luo, M., et al. (2008). An NADPH sensor protein (HSCARG) down-regulates nitric oxide synthesis by association with argininosuccinate synthetase and is essential for epithelial cell viability. J. Biol. Chem. 283, 11004-11013. doi: 10.1074/jbc.m7086 97200

Zhong, X., Chung, A. C., Chen, H.-Y., Meng, X.-M., and Lan, H. Y. (2011). Smad3mediated upregulation of miR-21 promotes renal fibrosis. J. Am. Soc. Nephrol. 22, 1668-1681. doi: 10.1681/asn.2010111168

Zhu, G.-H., Li, R., Zeng, Y., Zhou, T., Xiong, F., and Zhu, M. (2018). MicroRNA142-3p inhibits high-glucose-induced endothelial-to-mesenchymal transition through targeting TGF- $\beta 1 /$ Smad pathway in primary human aortic endothelial cells. Int. J. Clin. Exp. Pathol. 11:1208.

Conflict of Interest: The authors declare that the research was conducted in the absence of any commercial or financial relationships that could be construed as a potential conflict of interest.

Copyright (c) 2021 Giordo, Ahmed, Allam, Abusnana, Pappalardo, Nasrallah, Mangoni and Pintus. This is an open-access article distributed under the terms of the Creative Commons Attribution License (CC BY). The use, distribution or reproduction in other forums is permitted, provided the original author(s) and the copyright owner(s) are credited and that the original publication in this journal is cited, in accordance with accepted academic practice. No use, distribution or reproduction is permitted which does not comply with these terms. 